\title{
The combinatorics of the HMZ operators applied to Schur functions
}

\author{
Jeffrey B. Remmel and Meesue Yoo
}

\begin{abstract}
Haglund, Morse, and Zabrocki [12] introduced a family of symmetric function operators $\left\{\mathbb{B}_{m}\right\}_{m \geq 1}$ and $\left\{\mathbb{C}_{m}\right\}_{m \geq 1}$ which are closely related to operators of Jing [18]. Hanglund, Morse, and Zabrocki used these operators to refine the shuffle conjecture of Haglund, Haiman, Loehr, Remmel and Ulyanov [9] which gives a combinatorial interpretation of the coefficient of the monomial symmetric function in the Frobenius image of the character generating function of the ring of diagonal harmonics. In this paper, we give combinatorial interpretations of the coefficients that arise in Schur function expansion of $\mathbb{B}_{m} s_{\lambda}[X]$ and $\mathbb{C}_{m} s_{\lambda}[X]$ where $s_{\lambda}[X]$ is the Schur function associated to the partition $\lambda$. We then use such combinatorial interpretations to give a new recursion for the Kostka-Foulkes polynomials $K_{\lambda, \mu}(q)$.
\end{abstract}

AMS 2010 subJeCt CLASSIFICATIONS: Primary 05E05; secondary 05E10. KEYWORDS AND PHRASES: HMZ operators, Schur function expansion, plethysm, diagonal harmonics, cocharge.

Dedicated to Adriano Garsia on the occasion of his 84-th birthday.

\section{Introduction}

In [12], Haglund, Morse and Zabrocki introduced two new operators on symmetric functions which we call HMZ operators. In plethystic notation, these operators when applied to any symmetric function $P[X]$ are defined by

$$
\begin{aligned}
& \mathbb{B}_{m} P[X]=\left.P\left[X+\epsilon \frac{(1-q)}{z}\right] \Omega[-\epsilon z X]\right|_{z^{m}} \text { and } \\
& \mathbb{C}_{m} P[X]=\left.\left(-\frac{1}{q}\right)^{m-1} P\left[X-\frac{1-1 / q}{z}\right] \Omega[z X]\right|_{z^{m}}
\end{aligned}
$$

where $X=x_{1}+x_{2}+\cdots, \Omega[z X]=\prod_{i} \frac{1}{1-z x_{i}}$, and $\Omega[-\epsilon z X]=\prod_{i}\left(1+z x_{i}\right)$. 
Haglund, Morse and Zabrocki conjectured that these operators play an important role in the study of the combinatorics of the ring $R_{n}$ of coinvariants for the diagonal action of the symmetric group $S_{n}$ on $\mathbb{C}^{n} \oplus \mathbb{C}^{n}$. In other words, let

$$
R_{n}=\mathbb{C}[\mathbf{x}, \mathbf{y}] / I,
$$

where $\mathbb{C}[\mathbf{x}, \mathbf{y}]=\mathbb{C}\left[x_{1}, y_{1}, \ldots, x_{n}, y_{n}\right]$ is the ring of polynomial functions on $\mathbb{C}^{n} \oplus \mathbb{C}^{n}$, the symmetric group acts "diagonally" (i.e., permuting the $x$ and $y$ variables simultaneously), and the ideal $I=\left((\mathbf{x}, \mathbf{y}) \cap \mathbb{C}[\mathbf{x}, \mathbf{y}]^{S_{n}}\right)$ is generated by all $S_{n}$-invariant polynomials without a constant term. The $S_{n}$ action respects the double grading

$$
R_{n}=\bigoplus_{r, s}\left(R_{n}\right)_{r, s}
$$

given by the $x$ and $y$ degrees.

A formula for the character of $R_{n}$ as a doubly graded $S_{n}$ module was conjectured in [6] and proved in [17]. The formula expresses the character in terms of Macdonald polynomials as follows. Let $F$ denote the Frobenius characteristic: the linear map from $S_{n}$ characters to symmetric functions that sends the irreducible character $\chi^{\lambda}$ to the Schur function $s_{\lambda}[X]$. Encoding the graded character of $R_{n}$ by means of its Frobenius series

$$
\mathcal{F}_{R_{n}}(X ; q, t)=\sum_{r, s} q^{r} t^{s} F \operatorname{char}\left(R_{n}\right)_{r, s},
$$

its value is given by the following theorem.

Theorem 1.1. [17] Let $\nabla$ be the linear operator defined in terms of the modified Macdonald symmetric functions $\tilde{H}_{\mu}(X ; q, t)$ by

$$
\nabla \tilde{H}_{\mu}=t^{n(\mu)} q^{n\left(\mu^{\prime}\right)} \tilde{H}_{\mu},
$$

where $\mu$ is a partition of $n, \mu^{\prime}$ is its conjugate and $n(\mu)=\sum_{i}(i-1) \mu_{i}$. Then we have

$$
\mathcal{F}_{R_{n}}(X ; q, t)=\nabla e_{n}[X],
$$

where $e_{n}[X]$ is the nth elementary symmetric function. 
The combinatorics of the HMZ operators applied to Schur functions 403

Thanks to earlier results of Garsia and Haiman [6], Theorem 1.1 has combinatorially interesting consequences. Notably, the dimension of $R_{n}$ is given by

$$
\operatorname{dim}_{\mathbb{C}} R_{n}=(n+1)^{(n-1)},
$$

and that of its subspace $R_{n}^{\epsilon}$ of $S_{n}$-antisymmetric elements is given by

$$
\operatorname{dim}_{\mathbb{C}} R_{n}^{\epsilon}=C_{n}=\frac{1}{n+1}\left(\begin{array}{c}
2 n \\
n
\end{array}\right),
$$

the $n$-th Catalan number. These and other results elaborated in $[6,14,17]$ suggest that it should be possible to understand $\nabla e_{n}[X]$ in more combinatorial terms. A first step in this direction was taken by Garsia and Haglund $[4,5]$, who gave an explicit combinatorial formula for the Hall inner product

$$
C_{n}(q, t)=\left\langle\nabla e_{n}, e_{n}\right\rangle,
$$

which by Theorem 1.1 and equation (1.9) is a $q, t$-analog of the Catalan number $C_{n}(1,1)=C_{n}$. Building on the Garsia-Haglund formula, Haglund and Loehr [11] conjectured a combinatorial formula for the Hilbert series of $R_{n}$. By Theorem 1.1, this Hilbert series is given by

$$
\mathcal{H}_{n}(q, t)=\left\langle\nabla e_{n}, e_{1}^{n}\right\rangle=\sum_{r, s} q^{r} t^{s} \operatorname{dim}\left(R_{n}\right)_{r, s} .
$$

By [6], it was known that $\mathcal{H}_{n}(1, t)$ is a generating function enumerating parking functions according $t^{\text {area }(f)}$ where area $(f)$ is the number of cells that lie between the underlying Dyck path of the parking function $f$ and the diagonal. The Haglund-Loehr conjecture interprets $\mathcal{H}_{n}(q, t)$ as a bivariate generating function enumerating parking functions by area together with another statistic dinv which counts certain kinds of inversions (see [11]).

Later Haglund, Haiman, Loehr, Remmel and Uylanov [9] conjectured that if $h_{\lambda}[X]$ is the homogeneous symmetric function and $e_{\mu}[X]$ is the elementary symmetric function indexed by partitions $\lambda$ and $\mu$, then

$$
\left\langle\nabla e_{n}[X], h_{\lambda}[X] e_{\mu}[X]\right\rangle=\sum_{f \in \mathcal{P F}_{\lambda, \mu}} t^{\operatorname{area}(f)} q^{\operatorname{dinv}(f)}
$$

where $\mathcal{P} \mathcal{F}_{\lambda, \mu}$ is certain set of parking functions that depend on $\lambda$ and $\mu$. Their conjecture, which is now referred to as the shuffle conjecture, gener- 
alized the Garsia-Haglund formula for $C_{n}(q, t)$, the Haglund-Loehr conjecture for $\mathcal{H}_{n}(q, t)$, and a conjecture in [3] expressing $\left\langle\nabla e_{n}, h_{d} e_{n-d}\right\rangle$ in terms of Schröder paths which was proved by Haglund in [10].

The operator $\nabla$ described in Theorem 1.1 was introduced by F. Bergeron, Garsia, Haiman and Tesler [2]. When $\nabla$ is applied to various bases of symmetric functions, it encodes a lot of information about $q$, $t$-analogues of combinatorial objects such as lattice paths and parking functions. See [20, Table 1] where Loehr and Warrington provide a summary of such results. This paper is motivated by some recent progress in this area. First, Loehr and Warrington [20] gave a conjectured combinatorial interpretation for the coefficients that arise in the Schur function expansion of $\nabla s_{\lambda}[X]$ in terms of $q, t$-counting certain collections of nested Dyck paths depending on $\lambda$. N. Bergeron, Descouens and Zabrocki [1] studied the combinatorics of $\nabla$ applied to the $k$-Schur functions $s_{1^{n}}^{(k)}[X ; q]$ which is equal to the modified Hall-Littlewood polynomials when $\mu=\left(k^{\left\lfloor\frac{n}{k}\right\rfloor}, n \bmod k\right)$. Based on their conjecture on the coefficient of $s_{1^{n}}[X]$ in $\nabla s_{1^{n}}^{(k)}[X ; q]$, Haglund, Morse and Zabrocki [12] generalized the problem and conjectured the combinatorics of $\nabla$ applied to Hall-Littlewood symmetric functions indexed by compositions. In particular, Haglund, Morse and Zabrocki [12] refined the shuffle conjecture of [9]. That is, they conjectured that for any composition $p=\left(p_{1}, \ldots, p_{k}\right)$ of $n, \nabla \mathbb{C}_{p_{1}} \cdots \mathbb{C}_{p_{k}}(1)$ can be interpreted as $\sum_{f \in \mathcal{P} \mathcal{F}_{p}} t^{\operatorname{area}(f)} q^{\operatorname{din} v(f)}$ where $\mathcal{P} \mathcal{F}_{p}$ is the set of parking functions which hit the diagonal at exactly points $1,1+p_{1}, 1+p_{1}+p_{2}, \ldots, 1+p_{1}+\cdots+p_{k-1}, n$ and $\nabla \mathbb{B}_{p_{1}} \cdots \mathbb{B}_{p_{k}}(1)$ can be interpreted as $\sum_{f \in \mathcal{P} \mathcal{F}_{\bar{p}}} t^{\operatorname{area}(f)} q^{\operatorname{din} v(f)+\operatorname{dof} f_{p}(f)}$ where $\mathcal{P} \mathcal{F}_{\bar{p}}$ is the set of parking functions which hit the diagonal at least at the points $1,1+p_{1}, 1+p_{1}+$ $p_{2}, \ldots, 1+p_{1}+\cdots+p_{k-1}, n$, and $\operatorname{dof} f_{p}(f)$ is a specially defined $q$-statistic (see [12] for a detailed description).

The main goal of this paper is to give a combinatorial interpretation to the coefficients $b_{a, \lambda, \gamma}$ and $c_{a, \lambda, \gamma}$ that arise in the expansions

$$
\mathbb{B}_{a} s_{\lambda}[X]=\sum_{\gamma} b_{a, \lambda, \gamma} s_{\gamma}[X] \quad \text { and } \quad \mathbb{C}_{a} s_{\lambda}[X]=\sum_{\gamma} c_{a, \lambda, \gamma} s_{\gamma}[X]
$$

It then follows that

$$
\nabla \mathbb{B}_{a} s_{\lambda}[X]=\sum_{\gamma} b_{a, \lambda, \gamma} \nabla s_{\gamma}[X] \quad \text { and } \quad \nabla \mathbb{C}_{a} s_{\lambda}[X]=\sum_{\gamma} c_{a, \lambda, \gamma} \nabla s_{\gamma}[X]
$$

Thus using our results and the conjectures of Loehr and Warrington [20] on $\nabla s_{\lambda}$, we can produce combinatorial interpretations of $\nabla \mathbb{B}_{a} s_{\lambda}[X]$ and 

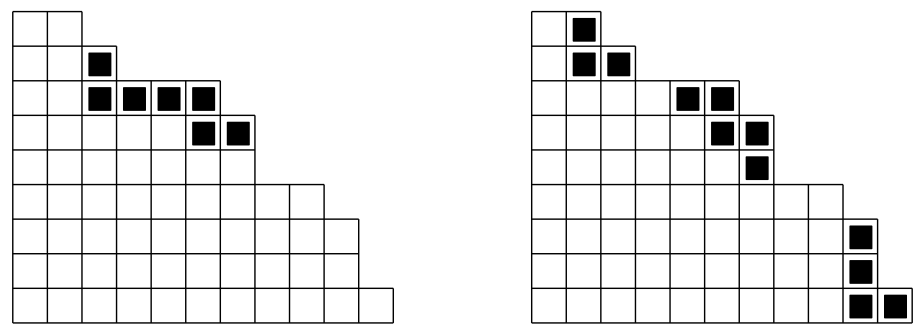

Figure 1: A rim hook (left) and a broken rim hook (right).

$\nabla \mathbb{C}_{a} s_{\lambda}[X]$. However, due to space considerations, we shall not study such combinatorial interpretations in this paper, but instead focus on the combinatorial interpretations of the coefficients $b_{a, \lambda, \gamma}$ and $c_{a, \lambda, \gamma}$.

To give an example of our results, we first need to establish some notation. We identify a partition $\lambda=\left(\lambda_{1} \geq \cdots \geq \lambda_{k}\right)$ with the Ferrers diagram of $\lambda$ (in French notation), i.e., the set of left justified rows of cells such that the $i$-th row has $\lambda_{i}$ cells, reading from bottom to top. For partitions $\lambda, \mu$, we write $\mu \subseteq \lambda$ whenever $\mu$ is contained within $\lambda$. In this case, we define the skew diagram $\lambda / \mu$ to be the set theoretic difference $\lambda-\mu$. A skew diagram forms a horizontal m-strip (vertical m-strip) if $|\lambda / \mu|=m$ and no cell of $\lambda / \mu$ lies immediately north (east) of another cell in $\lambda / \mu$.

A skew diagram $\lambda / \mu$ is a rim hook of $\lambda$ if $\lambda / \mu$ does not contain any $2 \times 2$ subdiagram and any two consecutive cells of $\lambda / \mu$ share an edge. A broken rim hook $(B R H)$ of $\lambda$ is a skew diagram $\lambda / \mu$ which is a union of rim hooks of $\lambda$. Figure 1 shows an example of a rim hook and a broken rim hook.

We shall show that $b_{a, \lambda, \gamma}=0$ unless $\gamma$ arises from $\lambda$ by first removing a broken rim hook $H$ from $\lambda$ and then adding a vertical strip $V$ in such a way that

1. all the cells of $V$ lie below all the cells of $H$,

2. if $H$ consists of rim hooks $\left(h_{1}, \ldots, h_{k}\right)$ reading from top to bottom, then $h_{1}$ must start in column 1 and for all $i=1, \ldots, k-1$, if the lowest cell of $h_{i}$ lies in column $s_{i}$, then the highest cell of $h_{i+1}$ must lie in column $s_{i}+1$, and

3. $|V|=a+|H|$.

In all the figures that follow, we shall always think of the cells of $H$ as being red and we shall indicate a red cell by placing an $r$ in that cell. Similarly, we shall always think of the cells of $V$ as colored blue and indicate a blue cell by placing a $b$ in that cell. Now suppose that $\gamma$ results from $\lambda$ by removing a broken rim hook $H$ of red cells and then adding a vertical strip $V$ 


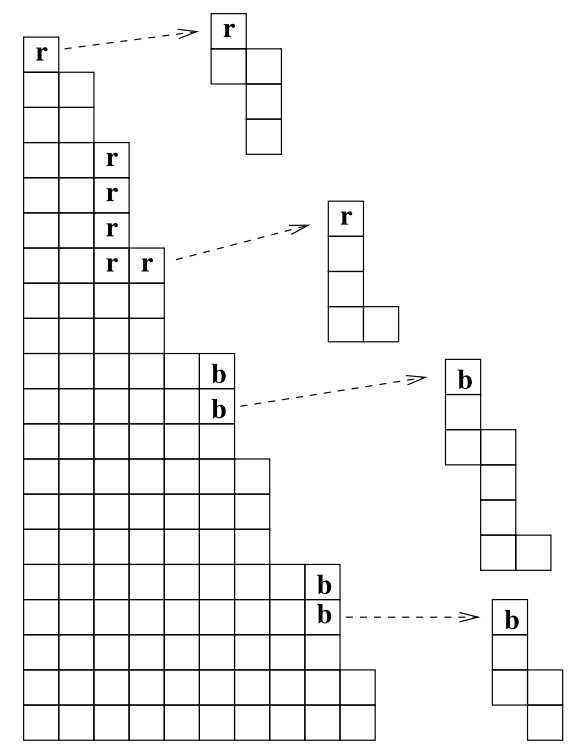

Figure 2: Connecting rim hooks.

of blue cells where $H$ and $V$ do not necessarily satisfy (1), (2), and (3). Then we know that cells in $\lambda-\gamma$ must be colored red and the cells in $\gamma-\lambda$ must be colored blue. However, $\lambda$ and $\gamma$ do not necessarily determine $H$ and $V$. That is, we shall need to consider what we call connecting rim hooks which consists of a rim hook of cells that starts at a colored cell or a blank cell and then consists a nonempty set of uncolored cells along the boundary of the Ferrers diagram up to the next cell which is either colored or is the last cell in the bottom row. For example, in Figure 2, we have pictured the four connecting rim hooks determined by $\lambda=\left(10^{2}, 9,8^{2}, 7^{3}, 6,5^{2}, 4^{3}, 3^{3}, 2^{2}, 1\right)$ and $\gamma=\left(10^{2}, 9^{3}, 7^{3}, 6^{3}, 4^{2}, 2^{6}\right)$. It is possible that some of the cells of any connecting rim hook could have been first removed since they were part of $H$ and then added back because they were part of $V$.

We shall see later that these connecting rim hooks play a crucial role in the computation of $b_{a, \lambda, \gamma}$ which has a rather simple combinatorial description. That is, suppose that $\gamma$ satisfies (1), (2), and (3). Let $u$ be the row of the lowest cell $c$ of $H$ and $v$ be the row which contains the first cell which lies below $c$ and strictly to the right of $c$. We let $R$ denote the set of red cells that do not lie in any connecting rim hooks. Then we shall prove that

$$
b_{a, \lambda, \gamma}=\left(q^{u-v}-1\right) q^{v-|V|}(-1)^{k-1} \prod_{d \in R} w_{B}(d)
$$


The combinatorics of the HMZ operators applied to Schur functions 407

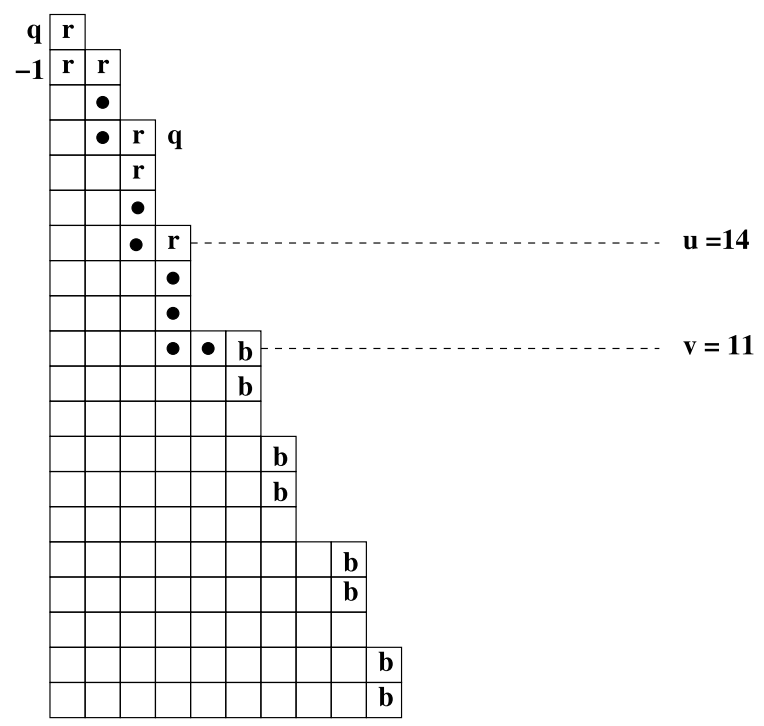

Figure 3: An example of $b_{a, \lambda, \gamma}$.

where

$$
w_{B}(c)= \begin{cases}-1 & \text { if } c \text { has a red cell immediately to its right } \\ q & \text { if } c \text { has a red cell immediately below it } \\ q-1 & \text { if otherwise }\end{cases}
$$

In the special case where $H$ is empty, then

$$
b_{a, \lambda, \gamma}=q^{\ell(\gamma)-|V|} .
$$

For example, in Figure 3, we have pictured such a $\gamma$ that could arise in the expansion of $\mathbb{B}_{2} s_{\lambda}[X]$ where $\lambda=\left(9^{3}, 8^{2}, 7,6^{3}, 5^{2}, 4^{3}, 3^{3}, 2^{2}, 1\right)$. We have put $r$ 's in the cells of $H, b$ 's in the cells of $V$, and we have put dots in the cells of the connecting rim hooks that start with a red cell. In this case $H=\left(h_{1}, h_{2}, h_{3}\right)$ consists of 3 rim hooks, $u=14$ and $v=11$. Note that $|H|=6$ and $|V|=8=2+|H|$. There are three red cells which are not part of connecting rim hooks and we have put $w_{B}(c)$ next to each of such cell $c$. Thus in this case,

$$
b_{a, \lambda, \gamma}=\left(q^{14-11}-1\right) q^{11-8}(-1)^{3-1} q^{2}=q^{5}\left(q^{3}-1\right) .
$$


Our results imply that in a number of special cases, it is quite easy to compute the expansion

$$
\mathbb{B}_{a} s_{\lambda}[X]=\sum_{\gamma} b_{a, \lambda, \gamma} s_{\gamma}[X]
$$

For example, if $a \geq \ell(\lambda)$, then it is not possible to construct a $\gamma$ where we first remove a non-empty broken rim hook $H$ that meets conditions (1), (2), and (3) so that

$$
\mathbb{B}_{a} s_{\lambda}[X]=\sum_{\gamma: \gamma / \lambda \text { is a vertical strip }} q^{\ell(\gamma)-a} s_{\gamma}[X] .
$$

The combinatorial interpretation of $c_{a, \lambda, \gamma}$ is a bit more complicated, but has a similar flavor. That is, we shall show that $c_{a, \lambda, \gamma}=0$ unless $\gamma$ arises from $\lambda$ by first removing a broken rim hook $H$ from $\lambda$ and then adding a horizontal strip $S$ such that

1. all the cells of $S$ lie above all the cells of $H$,

2. if $H$ consists of rim hooks $\left(h_{1}, \ldots, h_{k}\right)$ reading from bottom to top, then $h_{1}$ must start in row 1 and for all $i=1, \ldots, k-1$, if the top cell of $h_{i}$ lies in row $r_{i}$, then the bottom cell of $h_{i+1}$ must lie in row $r_{i}+1$, and

3. $|S|=a+|H|$.

Suppose that $\gamma$ satisfies (1), (2) and (3) and $u$ is the column of the highest red cell that was removed from $\lambda$, and $v$ is the column of the first corner cell to the left of the highest red cell of $\lambda$. Let $R$ denote the set of red cells that were removed from $\lambda$ which are not contained in any connecting rim hooks, and $p$ is the number of rim hooks that make the broken rim hook $\lambda / \gamma$. Then we shall prove that

$$
c_{a, \lambda, \gamma}(q)=(-1)^{p+a} \frac{q^{u-v}-1}{q^{u-|H|-2}(q-1)} \prod_{s \in R} w_{C}(s)
$$

where

$$
w_{C}(s)= \begin{cases}1 / q & \text { if } s \text { has a red cell to its right } \\ -1 & \text { if } s \text { has a red cell below it, and } \\ \frac{1-q}{q} & \text { if } s \text { is the lowest cell of a rim hook. }\end{cases}
$$

In the special case where $H$ is empty, then we simply have that

$$
c_{a, \lambda, \gamma}(q)=(-1)^{a-1}(1 / q)^{\gamma_{1}-1} .
$$


The combinatorics of the HMZ operators applied to Schur functions 409

The outline of this paper is as follows. In Section 2, we shall give the basic background on symmetric functions and plethystic notation that is needed for this paper. In Section 3, we shall summarize what is known about the HMZ operators $\mathbb{B}_{a}$ and $\mathbb{C}_{a}$. In Section 4 , we shall prove our combinatorial interpretation for the coefficients $b_{a, \lambda, \gamma}$. In Section 5, we shall prove our combinatorial interpretation for the coefficients $c_{a, \lambda, \gamma}$. The techniques that we use to prove our results in sections 4 and 5 are based on ideas due to Zabrocki [30] who analyzed the action of the Hall-Littlewood vertex operator on Schur functions and Remmel [25] who analyzed Macdonald's $D_{1}$ operator on Schur functions. Morris used different methods to compute closely related coefficients in [24]. Finally, in Section 6, we shall show how our results can be used to prove a new recursion for the $q$-Kostka-Foulkes polynomials $K_{\lambda, \mu}(q)$. Our recursion allows us to give an alternative method for computing $K_{\lambda, \mu}(q)$ which is different from the combinatorial definition of $K_{\lambda, \mu}(q)$ given by Lascoux and Schützenberger [19] in terms of the charge statistic.

\section{Basic definitions and notations}

\subsection{Basic objects}

A sequence $\alpha=\left(\alpha_{1}, \alpha_{2}, \ldots, \alpha_{r}\right)$ of positive integers is a composition of $n$ if $|\alpha|=\alpha_{1}+\alpha_{2}+\cdots+\alpha_{r}=n$. We use the notation $\alpha \mid=n$ to denote that $\alpha$ is a composition of $n$. We let $l(\alpha)$ denote $r$ which is the number of parts of $\alpha$. A composition of weakly decreasing parts is called a partition, i.e., $\lambda=\left(\lambda_{1}, \ldots, \lambda_{l(\lambda)}\right)$ with $\lambda_{1} \geq \lambda_{2} \geq \cdots \geq \lambda_{l(\lambda)}$ and $|\lambda|=\lambda_{1}+\cdots+\lambda_{l(\lambda)}=n$. We use the notation $\lambda \vdash n$ to denote that $\lambda$ is a partition of $n$.

For compositions (including partitions), we define

$$
n(\alpha)=\sum_{i=1}^{l(\alpha)}(i-1) \alpha_{i} .
$$

For a partition $\lambda$, a filling of $\lambda$ is a function $\sigma: \lambda \rightarrow[n]$ assigning integer entries to the cells of $\lambda$. A semi-standard Young tableau is a filling which is weakly increasing along each row of $\lambda$ and strictly increasing up each column. A semi-standard Young tableau is standard if it is a bijection from $\lambda$ to $[n]=\{1,2, \ldots, n\}$. For a partition $\lambda$ of $n$ and a composition $\nu$ of $n$, we define

$$
\begin{aligned}
\operatorname{SSYT}(\lambda) & =\{\text { semi-standard Young tableau } T: \lambda \rightarrow \mathbb{N}\}, \\
\operatorname{SSYT}(\lambda, \nu) & =\left\{\operatorname{SSYT} T: \lambda \rightarrow \mathbb{N} \text { with entries } 1^{\nu_{1}}, 2^{\nu_{2}}, \ldots\right\}, \\
\operatorname{SYT}(\lambda) & =\{\operatorname{SSYT} T: \lambda \stackrel{\sim}{\rightarrow}[n]\}=\operatorname{SSYT}\left(\lambda, 1^{n}\right) .
\end{aligned}
$$

For $T \in \operatorname{SSYT}(\lambda, \nu)$, we say $T$ is a $\operatorname{SSYT}$ of shape $\lambda$ and weight $\nu$. 


\subsection{Symmetric functions}

We shall mainly follow the notations of [23] for symmetric functions. We let $\Lambda^{n}$ denote the set of all homogeneous functions of degree $n$ over $\mathbb{Q}$ and $\Lambda=\Lambda^{1} \oplus \Lambda^{2} \oplus \cdots$.

Let $\lambda \vdash n$ be a partition of $n$. The elementary symmetric functions $e_{\lambda}$ are defined by setting $e_{\lambda}=\prod_{i} e_{\lambda_{i}}$ where $e_{0}=1$ and $e_{n}=\sum_{1 \leq i_{1}<\cdots<i_{n}} x_{i_{1}} \cdots x_{i_{n}}$ for $n \geq 1$. The complete homogeneous symmetric functions $h_{\lambda}$ are defined by setting $h_{\lambda}=\prod_{i} h_{\lambda_{i}}$ where $h_{0}=1$ and $h_{n}=\sum_{1<i_{1} \leq \cdots<i_{n}} x_{i_{1}} \cdots x_{i_{n}}$ for $n \geq 1$. The power sum symmetric functions $p_{\lambda}$ are defined by setting $p_{\lambda}=\prod_{i} p_{\lambda_{i}}$ where $p_{0}=1$ and $p_{n}=\sum_{i} x_{i}^{n}$ for $n \geq 1$. The skew Schur function $s_{\lambda / \mu}$ can be defined algebraically by

$$
s_{\lambda / \mu}=\operatorname{det}\left[h_{\lambda_{i}+i-\left(\mu_{j}+j\right)}\right]_{1 \leq i, j \leq n} .
$$

We also note that the skew Schur functions $s_{\lambda / \nu}$ are defined by the following property that for any $f \in \Lambda$,

$$
\left\langle f s_{\nu}, s_{\lambda}\right\rangle=\left\langle f, s_{\lambda / \nu}\right\rangle
$$

where $\langle$,$\rangle is the usual scalar product on \Lambda$ such that $\left\langle s_{\lambda}, s_{\mu}\right\rangle=\chi(\lambda=\mu)$. We let $\omega$ is an involution on symmetric functions such that $\omega\left(p_{\lambda}[X]\right)=$ $(-1)^{|\lambda|-l(\lambda)} p_{\lambda}[X], \omega\left(e_{n}[X]\right)=h_{n}[X]$ and $\omega\left(s_{\lambda}[X]\right)=s_{\lambda^{\prime}}[X]$.

Next we shall briefly review plethystic notation. Let $E=E\left(t_{1}, t_{2}, \ldots\right)$ be a formal Laurent series with rational coefficients in $t_{1}, t_{2}, \ldots$ We define the plethystic substitution $p_{k}[E]$ by replacing each $t_{i}$ in $E$ by $t_{i}^{k}$, i.e.,

$$
p_{k}[E]:=E\left(t_{1}^{k}, t_{2}^{k}, \ldots\right) .
$$

For any partition $\lambda=\left(\lambda_{1}, \ldots, \lambda_{k}\right)$ of $n$, we let $p_{\lambda}[E]=\prod_{i=1}^{k} p_{\lambda_{i}}[E]$. If $F=F\left(t_{1}, t_{2}, \ldots\right)$ is another formal Laurent series with rational coefficients in $t_{1}, t_{2}, \ldots$, then

(a) $p_{k}[E+F]=p_{k}[E]+p_{k}[F]$,

(b) $p_{k}[-E]=-p_{k}[E]=(-1)^{k}\left(\omega p_{k}\right)[E]$, and

(c) $p_{k}[E F]=p_{k}[E] p_{k}[F]$.

Then for any arbitrary symmetric function $f$, we define $f[E]$ as $\sum_{\lambda} c_{\lambda} p_{\lambda}[E]$ if $f=\sum_{\lambda} c_{\lambda} p_{\lambda}$.

Note that if $X=x_{1}+x_{2}+\cdots$,

$$
p_{k}[X]=\sum_{i \geq 1} x_{i}^{k}=p_{k}\left(x_{1}, x_{2}, \ldots\right) .
$$


The combinatorics of the HMZ operators applied to Schur functions 411

It then easily follows that for any $f \in \Lambda$,

$$
f[X]=f\left(x_{1}, x_{2}, \ldots\right) .
$$

For this reason, we consider this operation as a kind of substitution. In plethystic expression, $X$ stands for $x_{1}+x_{2}+\cdots$ so that $f[X]$ is the same as $f(X)=f\left(x_{1}, x_{2}, \ldots\right)$. See [22] for a fuller account.

Let $f$ be a symmetric function which is homogeneous of degree $d$. Then we have

$$
f[t X]=t^{d} f[X] \quad \text { and } \quad f[-X]=(-1)^{d} \omega f[X] .
$$

There is a special symbol $\epsilon$ which will represent a negative one but behaves differently than a negative symbol. That is, we define

$$
f[\epsilon X]=(-1)^{d} f[X] \quad \text { and } \quad f[-\epsilon X]=\omega(f[X]) .
$$

For any formal Laurent series $E=E\left(t_{1}, t_{2}, \ldots\right)$ with rational coefficients in $t_{1}, t_{2}, \ldots$, define

$$
\Omega[E]=e^{\sum_{k \geq 1} \frac{p_{k}[E]}{k}} .
$$

Then for $X=x_{1}+x_{2}+\cdots$ and $Y=y_{1}+y_{2}+\cdots$, we have the following identities

$$
\begin{aligned}
\Omega[X+Y] & =\Omega[X] \Omega[Y], \\
\Omega[X-Y] & =\Omega[X] / \Omega[Y], \\
\Omega[X] & =\prod_{i} \frac{1}{1-x_{i}}=\sum_{n \geq 0} h_{n}[X], \quad \text { and } \\
\Omega[-X] & =\prod_{i}\left(1-x_{i}\right)=\sum_{n \geq 0}(-1)^{n} e_{n}[X] .
\end{aligned}
$$

Next we note the following well known theorem from the theory of $\Lambda$-rings. For proofs, see [28, 22].

Theorem 2.1. For $X=x_{1}+x_{2}+\cdots$ and $Y=y_{1}+y_{2}+\cdots$,

$$
\begin{aligned}
s_{\mu / \lambda}[X+Y] & =\sum_{\lambda \subseteq \delta \subseteq \mu} s_{\mu / \delta}[X] s_{\delta / \lambda}[Y], \\
s_{\mu / \lambda}[-X] & =(-1)^{\left|\mu^{\prime} / \lambda^{\prime}\right|} s_{\mu^{\prime} / \lambda^{\prime}}[X], \\
s_{\mu / \lambda}[X-Y] & =\sum_{\lambda \subseteq \delta \subseteq \mu} s_{\mu / \delta}[X](-1)^{|\delta / \lambda|} s_{\delta^{\prime} / \lambda^{\prime}}[Y] .
\end{aligned}
$$


We will consider the modified Macdonald polynomials $\tilde{H}_{\mu}[X ; q, t]$ which are related to the integral form Macdonald polynomials $J_{\mu}[X ; q, t][23]$ by

$$
\tilde{H}_{\mu}[X ; q, t]=t^{n(\mu)} J_{\mu}\left[\frac{X}{1-1 / t} ; q, 1 / t\right] .
$$

We also note that

$$
q^{n(\mu)} \tilde{H}_{\mu}[X ; 0,1 / q]=\sum_{\lambda} K_{\lambda \mu}(q) s_{\lambda}[X]
$$

where $K_{\lambda \mu}(q)$ is the Kostka-Foulkes polynomial. Lascoux and Schützenberger [19] found that

$$
K_{\lambda \mu}(q)=\sum_{T \in \operatorname{SSYT}(\lambda, \mu)} q^{\operatorname{ch}(T)}
$$

where $\mu=\left(\mu_{1} \geq \mu_{2} \geq \cdots \geq \mu_{\ell(\mu)}\right)$ and $\operatorname{ch}(T)$ is the charge statistic. As in [23, III.6], we can define the charge statistic as follows. First we consider words $w=a_{1} \ldots a_{n}$, where the $a_{i}$ 's are positive integers. We say that $w$ is a standard word if it is a rearrangement of $1,2, \ldots, n$. We say that $w$ is of type $\mu$ if $w$ has $\mu_{1} 1 \mathrm{~s}, \mu_{2} 2 \mathrm{~s}$, etc.

(1) Given a standard word, we attach an index to letters in $w$ as follows. First 1 has index 0 . Then if $r$ has index $i, r+1$ has index $i$ if $r+1$ lies to the right of $r$ in $w$ and $r+1$ has index $i+1$ if $r+1$ lies to the left of $r$ in $w$. In this case, the charge of $w, \operatorname{ch}(w)$, is defined to be the sum of the indices of the letters in $w$. For example, if $w=2143$, then using the subscripts to indicate the index associated with each letter we would have $w=2_{1} 1_{0} 4_{2} 3_{1}$ so that $\operatorname{ch}(w)=4$.

(2) Now if the type of $w$ is $\mu=\left(\mu_{1} \geq \mu_{2} \geq \cdots\right)$, then one extracts a sequence of standard words $w^{(1)}, w^{(2)}, \ldots$ as follows. For $w^{(1)}$, we read the word from left to right and choose the first 1 that occurs in $w$, then the first 2 to the right of the 1 that is chosen and so on. If at any stage, there is no $s+1$ to the right of chosen $s$, we go back to the beginning of the word and choose the first $s+1$, then the first $s+2$ to the right of that $s+1$ is chosen and so on. We then let $w^{(1)}$ consist of the letters chosen in the procedure in the same order that they occur in $w$. This procedure will select a standard word of length $\mu_{1}^{\prime}$, where $\mu^{\prime}=\left(\mu_{1}^{\prime} \geq \mu_{2}^{\prime} \geq \cdots\right)$ is the conjugate of $\mu$. Then we erase the letters of $w^{(1)}$ from $w$ and repeat the procedure on the remaining letters to obtain a standard subword $w^{(2)}$ of length $\mu_{2}^{\prime}$ and so on. For example, if $w=32214131$, then 
The combinatorics of the HMZ operators applied to Schur functions 413

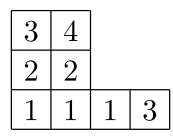

Figure 4: The word of a $T \in S S Y T((4,2,2),(3,2,2,1))$.

underlining the letters chosen in the first round of the procedure, we would get $w=3 \underline{2} 2 \underline{1} \underline{4} 1 \underline{3} 1$. Thus $w^{(1)}=2143$ and the remaining letters would be 3211 . It is then easy to check that $w^{(2)}=321$ and $w^{(3)}=1$. We then define the charge of $w$ by $\operatorname{ch}(w)=\sum_{i} \operatorname{ch}\left(w^{(i)}\right)$. For example, if $w=32214113$, then $\operatorname{ch}\left(w^{(1)}\right)=4, \operatorname{ch}\left(w^{(2)}\right)=3$, and $\operatorname{ch}\left(w^{(3)}\right)=0$ so that $\operatorname{ch}(w)=4+3+0=7$.

(3) Finally if $T \in \operatorname{SSYT}(\lambda, \mu)$, we construct the word of $T$ by reading the rows of $T$ from right to left and reading the rows from bottom to top. We then define the charge of $T$ by $\operatorname{ch}(T)=\operatorname{ch}(w(T))$. Thus for example, if $T$ is semi-standard Young tableaux of shape $\lambda=(4,2,2)$ and type $\mu=(3,2,2,1)$ pictured in Figure 4, then $w(T)=31112243$. Then one can check that $w^{(1)}=1243, w^{(2)}=312$, and $w^{(3)}=1$ so that

$$
\operatorname{ch}(T)=\operatorname{ch}(w(T))=\operatorname{ch}\left(w^{(1)}\right)+\operatorname{ch}\left(w^{(2)}\right)+\operatorname{ch}\left(w^{(3)}\right)=1+1+0=2 .
$$

\section{The symmetric operators $\mathbb{B}_{m}$ and $\mathbb{C}_{m}$}

Jing [18] defined symmetric function operators $\mathbb{H}_{m}$ for $m \geq 1$ by setting

$$
\mathbb{H}_{m} P[X]=\left.P\left[X-\frac{1-q}{z}\right] \Omega[z X]\right|_{z^{m}}
$$

for any symmetric function $P[X]$. These operators have the property that

$$
\mathbb{H}_{\mu_{1}} \mathbb{H}_{\mu_{2}} \cdots \mathbb{H}_{\mu_{l(\mu)}}(1)=q^{n(\mu)} \tilde{H}_{\mu}[X ; 0,1 / q]
$$

for $\mu$ a partition.

Haglund, Morse and Zabrocki [12] defined the following symmetric operators closely related to Jing's $\mathbb{H}_{m}$ operators defined in (3.1):

$$
\mathbb{B}_{m} P[X]=\left.P\left[X+\epsilon \frac{(1-q)}{z}\right] \Omega[-\epsilon z X]\right|_{z^{m}} \text { and }
$$




$$
\mathbb{C}_{m} P[X]=\left.\left(-\frac{1}{q}\right)^{m-1} P\left[X-\frac{1-1 / q}{z}\right] \Omega[z X]\right|_{z^{m}} .
$$

They also considered the symmetric functions defined by

$$
B_{\alpha}[X ; q]=\mathbb{B}_{\alpha_{1}} \mathbb{B}_{\alpha_{2}} \cdots \mathbb{B}_{\alpha_{l(\alpha)}}(1)
$$

and

$$
C_{\alpha}[X ; q]=\mathbb{C}_{\alpha_{1}} \mathbb{C}_{\alpha_{2}} \cdots \mathbb{C}_{\alpha_{l(\alpha)}}(1),
$$

for any composition $\alpha=\left(\alpha_{1}, \ldots, \alpha_{l(\alpha)}\right)$. These operators are related to the Jing's operators $\mathbb{H}_{m}$ by

$$
\mathbb{B}_{m}=\omega \mathbb{H}_{m} \omega \text { and } \quad \mathbb{C}_{m}=(-1 / q)^{m-1} \mathbb{H}_{m}^{q \rightarrow 1 / q}
$$

and so

$$
\mathbb{C}_{m}=(-1 / q)^{m-1} \omega \mathbb{B}_{m}^{q \rightarrow 1 / q} \omega
$$

Thus, by (3.2), for a partition $\mu$, we have the relations

$$
\begin{aligned}
& B_{\mu}[X ; q]=q^{n(\mu)} \omega \tilde{H}_{\mu}[X ; 0,1 / q] \text { and } \\
& C_{\mu}[X ; q]=q^{-n(\mu)}(-1 / q)^{|\mu|-l(\mu)} \tilde{H}_{\mu}[X ; 0, q] .
\end{aligned}
$$

Note that (3.3) and (3.4) imply that

$$
B_{\mu}[X ; q]=\omega \sum_{\lambda} K_{\lambda \mu}(q) s_{\lambda}[X]=\sum_{\lambda} K_{\lambda \mu}(q) s_{\lambda^{\prime}}[X]
$$

and

$$
C_{\mu}[X ; q]=(-1)^{|\mu|-l(\mu)}(1 / q)^{|\mu|-l(\mu)+n(\mu)} \sum_{\lambda} \tilde{K}_{\lambda \mu}(q) s_{\lambda}[X]
$$

where $\tilde{K}_{\lambda \mu}(q)=q^{n(\mu)} K_{\lambda \mu}(1 / q)=\sum_{T \in \operatorname{SSYT}(\lambda, \mu)} q^{\operatorname{coch}(T)}$ for $\operatorname{coch}(T)=n(\mu)-$ $\operatorname{ch}(T)$.

To manipulate the symmetric functions $B_{\alpha}[X ; q]$ and $C_{\alpha}[X ; q]$, Haglund, Morse and Zabrocki proved the following commutativity relations of $\mathbb{B}$ and $\mathbb{C}$ operators.

Theorem 3.1. [12] For $a, b \in \mathbb{Z}$, we have

$$
\mathbb{B}_{b} \mathbb{B}_{a}+\mathbb{B}_{a-1} \mathbb{B}_{b+1}=q\left(\mathbb{B}_{a} \mathbb{B}_{b}+\mathbb{B}_{b+1} \mathbb{B}_{a-1}\right),
$$


The combinatorics of the HMZ operators applied to Schur functions 415

$$
q\left(\mathbb{C}_{b} \mathbb{C}_{a}+\mathbb{C}_{a-1} \mathbb{C}_{b+1}\right)=\mathbb{C}_{a} \mathbb{C}_{b}+\mathbb{C}_{b+1} \mathbb{C}_{a-1} .
$$

For $a+b>0$,

$$
\mathbb{B}_{a} \mathbb{C}_{b}=q \mathbb{C}_{b} \mathbb{B}_{a} .
$$

In [6], Garsia and Haglund proved that their $q, t$-analogue of the Catalan number, $C_{n}(q, t)$, is a polynomial with positive integer coefficients by using the following identity

$$
C_{n}(q, t)=\left.\nabla e_{n}[X]\right|_{s_{1} n} .
$$

They defined the special symmetric function element $E_{n, k}[X ; q]$ by the following identity

$$
e_{n}\left[X \frac{1-z}{1-q}\right]=\sum_{k=1}^{n} \frac{(z ; q)_{k}}{(q ; q)_{k}} E_{n, k}[X ; q]
$$

and they proved that the coefficient of $e_{n}[X]$ in $\nabla\left(E_{n, k}[X ; q]\right) q, t$-enumerates the Dyck paths which touch the diagonal $k$ times.

Haglund, Morse and Zabrocki [12] conjectured combinatorial formulas for the monomial expansions of $\nabla\left(B_{\alpha}[X ; q]\right)$ and $\nabla\left(C_{\alpha}[X ; q]\right)$ which give the coefficient of $e_{n}[X]$ in $\nabla\left(B_{\alpha}[X ; q]\right)$ and $\nabla\left(C_{\alpha}[X ; q]\right)$. According to their conjectured formulas, we have

$$
\left\langle e_{n}[X], \nabla\left(E_{n, k}[X ; q]\right)\right\rangle=\sum_{\alpha \models n, l(\alpha)=k}\left\langle e_{n}[X], \nabla\left(C_{\alpha}[X ; q]\right)\right\rangle .
$$

They also showed that this identity holds in more generality by expanding $E_{n, k}[X ; q]$ in terms of $C_{\alpha}[X]$, namely,

$$
E_{n, k}[X ; q]=\sum_{\alpha \models n, l(\alpha)=k} C_{\alpha}[X ; q] .
$$

This identity is from the following simple expression for $e_{n}[X]$ in terms of $C_{\alpha}[X]$.

Proposition 3.2. [12]

$$
e_{n}[X]=\sum_{\alpha \models n} C_{\alpha}[X ; q] .
$$

There is a slight generalization of Proposition 3.2. 
Proposition 3.3. [12] For $0 \leq k<n$,

$$
s_{\left(n-k, 1^{k}\right)}[X]=(-q)^{n-k-1} \sum_{\substack{\alpha=n \\ \alpha_{1} \geq n-k}} C_{\alpha}[X ; q] .
$$

The main goal of this paper is to find combinatorial interpretations for the coefficients that arise in the Schur function expansion of $\mathbb{B}_{m} s_{\lambda}[X]$ and $\mathbb{C}_{m} s_{\lambda}[X]$. We shall also give simple recursions for the expansion of $\mathbb{B}_{m} e_{\lambda}[X]$ in terms of the elementary symmetric functions and $\mathbb{C}_{m} h_{\lambda}[X]$ in terms of the homogeneous symmetric functions. These recursions can be used to give alternative proofs of many identities involving the operations $\mathbb{B}_{m}$ and $\mathbb{C}_{m}$ described above.

\section{Computing $\mathbb{B}_{m} s_{\lambda}[X]$}

By the definition of $\mathbb{B}_{m}$,

$$
\begin{aligned}
& \mathbb{B}_{m} s_{\lambda / \nu}[X] \\
& =\left.s_{\lambda / \nu}\left[X+\epsilon \frac{(1-q)}{z}\right] \Omega[-\epsilon z X]\right|_{z^{m}} \\
& =\left.\sum_{\nu \subseteq \mu \subseteq \lambda} s_{\mu / \nu}[X] s_{\lambda / \mu}\left[\epsilon \frac{1-q}{z}\right] \sum_{n \geq 0} z^{n} \omega\left(h_{n}[X]\right)\right|_{z^{m}} \\
& =\left.\sum_{\nu \subseteq \mu \subseteq \lambda} s_{\mu / \nu}[X]\left(\frac{-1}{z}\right)^{|\lambda / \mu|} s_{\lambda / \mu}[1-q] \sum_{n \geq 0} z^{n} e_{n}[X]\right|_{z^{m}} \\
& =\sum_{\nu \subseteq \mu \subseteq \lambda} s_{\mu / \nu}[X]\left(\frac{-1}{z}\right)^{|\lambda / \mu|} \\
& \times\left.\left(\sum_{\mu \subseteq \beta \subseteq \lambda} s_{\lambda / \beta}[1](-1)^{|\beta / \mu|} s_{\beta^{\prime} / \mu^{\prime}}[q]\right) \sum_{n \geq 0} z^{n} e_{n}[X]\right|_{z^{m}} \\
& =\sum_{\nu \subseteq \mu \subseteq \lambda} s_{\mu / \nu}[X] \underbrace{\left(\sum_{\mu \subseteq \beta \subseteq \lambda}(-1)^{|\lambda / \beta|} s_{\lambda / \beta}[1] s_{\beta^{\prime} / \mu^{\prime}}[q]\right)}_{(*)} e_{m+|\lambda / \mu|}[X] .
\end{aligned}
$$


The combinatorics of the HMZ operators applied to Schur functions 417

We consider a combinatorial interpretation of $(*)$ in $(4.2)$ which is

$$
(-1)^{\lambda / \mu} s_{\lambda / \mu}[1-q]=\sum_{\mu \subseteq \beta \subseteq \lambda}(-1)^{|\lambda / \beta|} s_{\lambda / \beta}[1] s_{\beta^{\prime} / \mu^{\prime}}[q] .
$$

Note that $s_{\lambda / \beta}[1]=0$ unless $\lambda / \beta$ is a horizontal strip and if $\lambda / \beta$ is a horizontal strip, then $s_{\lambda / \beta}[1]=1$. Similarly, $s_{\beta^{\prime} / \mu^{\prime}}[q]=0$ unless $\beta / \mu$ is a vertical strip and if it is a vertical strip, then $s_{\beta^{\prime} / \mu^{\prime}}[q]=q^{|\beta / \mu|}$. Thus we see that $s_{\mu / \nu}$ occurs in the expansion of $\mathbb{B}_{m} s_{\lambda / \nu}[X]$ only if $\mu / \nu$ arises from $\lambda$ by first removing a horizontal strip to obtain $\beta / \nu$ and then removing a vertical strip to obtain $\mu / \nu$ which implies that $\lambda / \mu$ is a broken rim hook (BRH). In such a situation, the cells of $\lambda / \beta$ have a weight of -1 which we indicate by placing a -1 in such cells. Similarly, the cells of $\beta / \mu$ have a weight of $q$ which we indicate by placing a $q$ in such cells. It follows that we must weight the cells in $\lambda / \mu$ by taking the product of the entries of $\lambda / \mu$ where

(i) if $s$ is a cell which has a cell in $\lambda / \mu$ to its right, then we place a -1 in it,

(ii) if $s$ is a cell which has a cell in $\lambda / \mu$ below it, then we place a $q$ in it, and

(iii) if $s$ is the lowest cell in one of the rim hooks of $\lambda / \mu$ so that it does not have a cell in $\lambda / \mu$ directly to its right or directly below it, then we can place either a $q$ or -1 in $s$.

It follows that

$$
\mathbb{B}_{m} s_{\lambda / \nu}[X]=\sum_{\substack{\nu \subseteq \mu \subseteq \lambda, \lambda / \mu \text { is a } \mathrm{BRH} \text { of } \lambda}} w_{B}(\lambda / \mu) s_{\mu / \nu}[X] e_{m+|\lambda / \mu|}[X]
$$

where

$$
w_{B}(\lambda / \mu)=\prod_{s \in \lambda / \mu} w_{B, \lambda / \mu}(s)
$$

and for each cell $s \in \lambda / \mu$,

(4.4) $w_{B, \lambda / \mu}(s)= \begin{cases}-1 & \text { if } s \text { has a cell of } \lambda / \mu \text { to its right } \\ q & \text { if } s \text { has a cell of } \lambda / \mu \text { below it, and } \\ q-1 & \text { if } s \text { is the lowest cell of a rim hook in } \lambda / \mu .\end{cases}$ 


\section{1. $\mathbb{B}_{m}$ applied to elementary symmetric basis $e_{\lambda}$}

Our first application of (4.3) is to derive a simple recursion for $\mathbb{B}_{m}$ applied to an elementary symmetric function $e_{\lambda}=e_{\lambda_{1}} e_{\lambda_{2}} \cdots e_{\lambda_{l(\lambda)}}$. That is, we have the recursion

$$
\mathbb{B}_{m}\left(e_{s} e_{\lambda}\right)=e_{s} \mathbb{B}_{m}\left(e_{\lambda}\right)+(q-1) \sum_{j=1}^{s} q^{j-1} e_{s-j} \mathbb{B}_{m+j}\left(e_{\lambda}\right)
$$

for any $\lambda \vdash n$ and $s \geq 1$. That is, since $e_{n}=s_{1^{n}}$, we can think of

$$
e_{\lambda}=s_{1^{\lambda_{1}}} s_{1^{\lambda_{2}}} \cdots s_{1^{l(\lambda)}},
$$

as a skew Schur function corresponding to $\prod_{i} s_{1^{\lambda_{i}}}$. It follows from (4.3) that

$$
\mathbb{B}_{m}\left(e_{\lambda}\right)=\left.\prod_{i=1}^{l(\lambda)}\left(e_{\lambda_{i}}+(q-1) \sum_{j=1}^{\lambda_{i}} \frac{q^{j-1}}{z^{j}} e_{\lambda_{i}-j}\right)\left(\sum_{n \geq 0} e_{n} z^{n}\right)\right|_{z^{m}}
$$

Hence

$$
\begin{aligned}
\mathbb{B}_{m}\left(e_{s} e_{\lambda}\right) & \left(e_{s}+(q-1) \sum_{j=1}^{s} \frac{q^{j-1}}{z^{j}} e_{s-j}\right) \\
& \times\left.\prod_{i=1}^{l(\lambda)}\left(e_{\lambda_{i}}+(q-1) \sum_{j=1}^{\lambda_{i}} \frac{q^{j-1}}{z^{j}} e_{\lambda_{i}-j}\right)\left(\sum_{n \geq 0} e_{n} z^{n}\right)\right|_{z^{m}} \\
= & \left.e_{s} \prod_{i=1}^{l(\lambda)}\left(e_{\lambda_{i}}+(q-1) \sum_{j=1}^{\lambda_{i}} \frac{q^{j-1}}{z^{j}} e_{\lambda_{i}-j}\right)\left(\sum_{n \geq 0} e_{n} z^{n}\right)\right|_{z^{m}} \\
& +(q-1) \sum_{j=1}^{s}\left[q^{j-1} e_{s-j} \prod_{i=1}^{l(\lambda)}\left(e_{\lambda_{i}}+(q-1) \sum_{j=1}^{\lambda_{i}} \frac{q^{j-1}}{z^{j}} e_{\lambda_{i}-j}\right)\right. \\
& \left.\times\left(\sum_{n \geq 0} e_{n} z^{n}\right)\right]||_{z^{m+j}}^{s}{ }_{j=1}^{s} q^{j-1} e_{s-j} \mathbb{B}_{m+j}\left(e_{\lambda}\right) . \\
= & e_{s} \mathbb{B}_{m}\left(e_{\lambda}\right)+(q-1) \sum_{j=1}
\end{aligned}
$$


The combinatorics of the HMZ operators applied to Schur functions 419

One can use (4.5) to give a simple inductive proof of the fact in Theorem 3.1 that

$$
\mathbb{B}_{b} \mathbb{B}_{a}+\mathbb{B}_{a-1} \mathbb{B}_{b+1}=q\left(\mathbb{B}_{a} \mathbb{B}_{b}+\mathbb{B}_{b+1} \mathbb{B}_{a-1}\right)
$$

by showing that both sides of (4.7) gives the same result when they are applied to any $e_{\lambda}$.

Note by (4.5), we have that

$$
\begin{aligned}
\mathbb{B}_{m}^{q \rightarrow 1 / q}\left(e_{s} e_{\lambda}\right) & =e_{s} \mathbb{B}_{m}^{q \rightarrow 1 / q}\left(e_{\lambda}\right)+\left(\frac{1}{q}-1\right) \sum_{j=1}^{s}\left(\frac{1}{q}\right)^{j-1} e_{s-j} \mathbb{B}_{m+j}^{q \rightarrow 1 / q}\left(e_{\lambda}\right) \\
& =e_{s} \mathbb{B}_{m}^{q \rightarrow 1 / q}\left(e_{\lambda}\right)+(1-q) \sum_{j=1}^{s}\left(\frac{1}{q}\right)^{j} e_{s-j} \mathbb{B}_{m+j}^{q \rightarrow 1 / q}\left(e_{\lambda}\right) .
\end{aligned}
$$

Applying the operator $(-1 / q)^{m-1} \omega$ to both sides of (4.8) and using (3.5), we obtain that

$$
\mathbb{C}_{m}\left(h_{s} h_{\lambda}\right)=h_{s} \mathbb{C}_{m}\left(h_{\lambda}\right)+(1-q) \sum_{j=1}^{s}(-1)^{j} h_{s-j} \mathbb{C}_{m+j}\left(h_{\lambda}\right) .
$$

One can use recursion (4.9) to give a simple inductive proof of the fact in Theorem 3.1 that

$$
q\left(\mathbb{C}_{b} \mathbb{C}_{a}+\mathbb{C}_{a-1} \mathbb{C}_{b+1}\right)=\mathbb{C}_{a} \mathbb{C}_{b}+\mathbb{C}_{b+1} \mathbb{C}_{a-1} .
$$

That is, one can easily show by induction on the number of parts of $\lambda$ that both sides of (4.10) give the same answer when applied to $h_{\lambda}$.

\section{2. $\mathbb{B}_{m}$ applied to Schur functions}

Next, we consider the Schur expansion of $\mathbb{B}_{m}$ applied to the Schur function $s_{\lambda}[X]$,

$$
\mathbb{B}_{m} s_{\lambda}[X]=\sum_{\substack{\mu \subseteq \lambda, \lambda / \mu \text { is a BRH of } \lambda}} w_{B}(\lambda / \mu) s_{\mu}[X] e_{m+|\lambda / \mu|}[X],
$$

where

$$
w_{B}(\lambda / \mu)=\prod_{s \in \lambda / \mu} w_{B, \lambda / \mu}(s),
$$


and for each cell $s \in \lambda / \mu$, the weight $w_{B, \lambda / \mu}(s)$ is as defined in (4.4). In (4.11), one can apply the Pieri rule to see that

$$
s_{\mu}[X] e_{m+|\lambda / \mu|}[X]=\sum_{\substack{\mu \subseteq \gamma, \gamma / \mu \text { is a } \\ \text { vertical strip of size } m+|\lambda / \mu|}} s_{\gamma}[X] .
$$

Thus

$$
\begin{aligned}
\mathbb{B}_{m} s_{\lambda}[X] & =\sum_{\mu \subseteq \lambda, \lambda / \mu \text { is a BRH of } \lambda} w_{B}(\lambda / \mu) s_{\mu}[X] e_{m+|\lambda / \mu|}[X] \\
& =\sum_{\substack{\mu \subseteq \lambda, \lambda / \mu \text { is a BRH of } \lambda \\
\mu \subseteq \gamma, \gamma / \mu \text { is a vertical strip of size } m+|\lambda / \mu|}} w_{B}(\lambda / \mu) s_{\gamma}[X] .
\end{aligned}
$$

Each $\gamma$ that appears on the right hand side of (4.12) can be constructed by first removing a broken rim hook $\lambda / \mu$ from $\lambda$ and then adding back a vertical strip $\gamma / \mu$ of size $|\lambda / \mu|+m$.

We shall think of the cells of the broken rim hook $\lambda / \mu$ as being colored red so we will put an $r$ in the cells corresponding to $\lambda / \mu$ and we shall think of the cells of the vertical strip $\gamma / \mu$ as being colored blue so we will put a $b$ in the cells corresponding to $\gamma / \mu$. Then in the right hand side of (4.12), notice that the red and blue cells completely determine $\lambda$ and $\gamma$, but there could be many $\mu$ 's that could give rise to the same $\lambda$ and $\gamma$. That is, the cells in the rim hooks connecting colored cells could be that some of these cells were originally removed from $\lambda$ to form $\mu$ and then added back to form $\gamma$. Figure 5 shows various possibilities of $\mu$ when $\lambda=\left(3^{2}, 2^{3}\right)$ and $\gamma=\left(4^{2}, 2^{2}, 1\right)$. Thus the final weight associated with $s_{\gamma}$ in (4.12) should be the sum of $w_{B}(\lambda / \mu)$ over all possible $\mu$ 's. We shall make the convention that the top colored cell, if there is any, is part of the connecting rim hook while the bottom colored cell, if it exists, is not part of the connecting rim hook. Nevertheless, the final weight associated with the connecting rim hook does depend on the colored square at the bottom. Thus when we talk about connecting rim hooks, it will be convenient to refer to the connecting rim hook by the top cell and
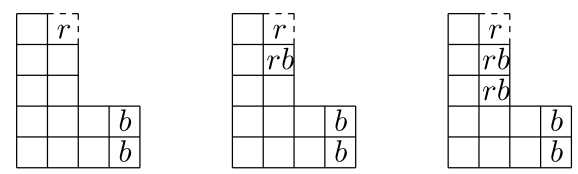

Figure 5: Possible $\mu$ 's for $\lambda=\left(3^{2}, 2^{3}\right)$ and $\gamma=\left(4^{2}, 2^{2}, 1\right)$. 
The combinatorics of the HMZ operators applied to Schur functions 421

the cell next to the bottom cell if it exists. Thus a $r-b$ connecting rim hook is a connecting rim hook that starts with a red cell and has its final cell connected to a blue cell, a blank- $b$ connecting is a connecting rim hook that starts with a blank cell and has its final cell connected to a blue cell, etc. This given, if we let the connecting rim hooks of $\lambda \cup \gamma$ be $H_{1}, \ldots, H_{k}$, and the red cells which are not part of any connecting rim hooks be $r_{1}, \ldots, r_{s}$, then the total weight associated with $s_{\gamma}[X]$ would be

$$
\left(\prod_{i=1}^{k} W_{B}\left(H_{i}\right)\right)\left(\prod_{i=1}^{s} w_{B, \lambda / \gamma}\left(r_{i}\right)\right),
$$

where $W_{B}\left(H_{i}\right)$ is the sum of all the possible weights that can occur in $H_{i}$ as $\mu$ varies in (4.12). For instance, in the case the connecting rim hook $H$ for Figure 5,

$$
W_{B}(H)=(q-1)+q(q-1)+q^{2}(q-1)=q^{3}-1 .
$$

In the following subsections, we shall calculate explicit formulas for the weights of all possible connecting rim hook shapes.

\subsection{Computing weights of connecting rim hooks}

We start by computing the weights of simple connecting rim hooks, namely when they are just a column or just a row.

Let $r V(L), b V(L)$ and $V(L)$ denote the connecting rim hooks of a column of $L$ squares with a red cell on the top, a blue cell on the top, and a blank cell on the top, respectively. Then we have the following lemma.

Lemma 4.1. For $L \geq 1$,

$$
\begin{aligned}
W_{B}(r V(L)) & =q^{L+1}-1, \\
W_{B}(b V(L)) & =q^{L}, \quad \text { and } \\
W_{B}(V(L)) & =q^{L} .
\end{aligned}
$$

Proof. We calculate $W_{B}(r V(L))$ first. If there are $k$ cells colored $r b$ (in other words, if they are removed from $\lambda$ first and added back to form $\gamma$ ) below the red cell on the top, then the weight would be $q^{k}(q-1)$. Note that $k$ can be any value from 0 to $L$. By adding the weights over all possible $k$ values, we get

$$
W_{B}(r V(L))=\sum_{k=0}^{L} q^{k}(q-1)
$$




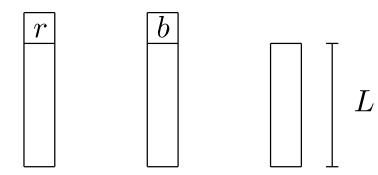

Figure 6: $r V(L), b V(L)$ and $V(L)$, from the left.

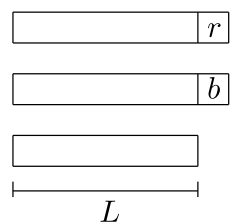

Figure 7: $H r(L), H b(L)$ and $H(L)$ from the top.

$$
\begin{aligned}
& =(q-1) \frac{q^{L+1}-1}{q-1} \\
& =q^{L+1}-1 .
\end{aligned}
$$

If a $b$ cell is on the top and there are $k r b$ cells below it, then the weight is $q^{k-1}(q-1)$ if $k \geq 1$ and the weight is 1 if $k=0$. Hence

$$
\begin{aligned}
W_{B}(b V(L)) & =1+\sum_{k=1}^{L} q^{k-1}(q-1) \\
& =1+(q-1) \frac{q^{L}-1}{q-1} \\
& =q^{L}
\end{aligned}
$$

The same computation shows that $W_{B}(V(L))=q^{L}$.

We let $H r(L), H b(L)$ and $H(L)$ denote the connecting rim hooks of one row with $L$ squares with a red cell, a blue cell, or nothing attached to its right, respectively. Then we have the following lemma.

Lemma 4.2. For $L \geq 1$,

$$
\begin{aligned}
W_{B}(H r(L)) & =0, \\
W_{B}(H b(L)) & =1, \quad \text { and } \\
W_{B}(H(L)) & =q .
\end{aligned}
$$


The combinatorics of the HMZ operators applied to Schur functions 423

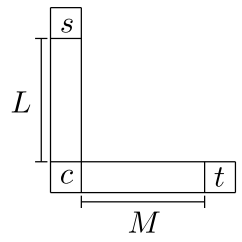

Figure 8: A hook shape connecting rim hook, $\operatorname{sVHt}(L, M)$.

Proof. We start by calculating $W_{B}(H r(L))$. Since the blue cells must form a vertical strip there are only two possible cases for $\operatorname{Hr}(L)$. That is, either we do nothing or we color the cell immediately to the left of the red cell in $r b$. Thus,

$$
W_{B}(H r(L))=(q-1)+(-1)(q-1)=0 .
$$

If blue cell is the last cell of the row, there can be no $r b$ cells so its weight is 1 .

If there is no colored cell at the right end of the row, then we can remove and add only one $r b$ cell at the right end, and this would give

$$
W_{B}(H(L))=1+(q-1)=q .
$$

Before we handle the case of general connecting rim hooks, we first need to compute the weights of connecting rim hooks of a hook shape.

Proposition 4.3. Let $\operatorname{sVHt}(L, M)$ denote a hook shape connecting rim hook connecting cells colored in $s$ and $t$ as in Figure 8. If $M \geq 1$, then

$$
W_{B}(\operatorname{sVHt}(L, M))= \begin{cases}W_{B}(s V(L)), & \text { if } t=b, \\ q W_{B}(s V(L)), & \text { if } t=b \text { lank } \\ 0, & \text { if } t=r .\end{cases}
$$

If $M=0$, then

$$
W_{B}(\operatorname{sVHt}(L, M))= \begin{cases}W_{B}(s V(L)), & \text { if } t=b \text { or blank } \\ -1, & \text { if } s=r, t=r \\ 0, & \text { if } s=\text { blank or } b, t=r,\end{cases}
$$

where $W_{B}(s V(L))$ is as defined in Lemma 4.1.

Proof. Let $c$ denote the left most corner cell in the bottom row. If $M \geq 1$ and $t=b$, then $c$ cannot be colored in $r b$ (i.e., removed and added back) since the blue cells form a vertical strip. Thus the weight of the hook $s V H b(L, M)$ 
would be consistent with the weight of a vertical strip $s V(L)$ as in Lemma 4.1. The same reasoning can be applied to the case when $M=0$ and $t=b$. If $t=r$ and $M \geq 1$, then again the cell $c$ cannot be colored $r b$ and there are only two possibilities for the horizontal part;

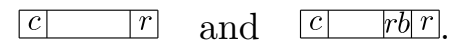

For the above two horizontal strips, the sum of possible weights are 0 by Lemma 4.2. Since the cell $c$ is uncolored, it separates the vertical strip $s V(L)$ and the horizontal strip $\operatorname{Hr}(M)$. Hence,

$$
W_{B}(s V H t(L, M))=W_{B}(s V(L)) W_{B}(H r(M))
$$

which is 0 since $W_{B}(\operatorname{Hr}(M))=0$. Similarly, if $M \geq 1$ and $t$ is blank, then there are only two possibilities for the horizontal part;

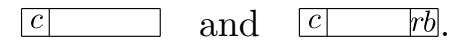

So, $W_{B}(H(M))=1+(q-1)=q$ and

$$
W_{B}(s V H t(L, M))=W_{B}(s V(L)) W_{B}(H(M)),
$$

where $W_{B}(s V(L))$ as described in Lemma 4.1.

Next we consider the case when $M=0$ and $t=r$. If $s=r$, and there are $k$ cells colored $r b$ below the top $r$ cell where $0 \leq k \leq L$, then the weight of $r V H t(L, 0)$ would be $q^{k}(q-1)$. However, in this case, we also have the possibility of coloring all the cells in $L$ plus cell $c$ with $r b$, and then the weight of the hook $r \operatorname{HHr}(L, 0)$ would be $-q^{L+1}$. Hence,

$$
\begin{aligned}
W_{B}(r V H r(L, 0)) & =\sum_{k=0}^{L} q^{k}(q-1)-q^{L+1} \\
& =-1 .
\end{aligned}
$$

If $s=b$ or blank, then in the case when there are no $r b$-colored cells in the vertical part, the weight of $s V H(L, 0) r$ is 1 . Hence,

$$
\begin{aligned}
W_{B}(s \operatorname{VHr}(L, 0)) & =1+\sum_{k=1}^{L} q^{k-1}(q-1)-q^{L} \\
& =0 .
\end{aligned}
$$

If $M=0$ and $t$ is blank, then since the hook $s V H(L, M)$ is just a vertical strip $s V(L)$, it has the same weight as $W_{B}(s V(L))$. 
The combinatorics of the HMZ operators applied to Schur functions 425

Table 1: Weights of connecting rim hooks

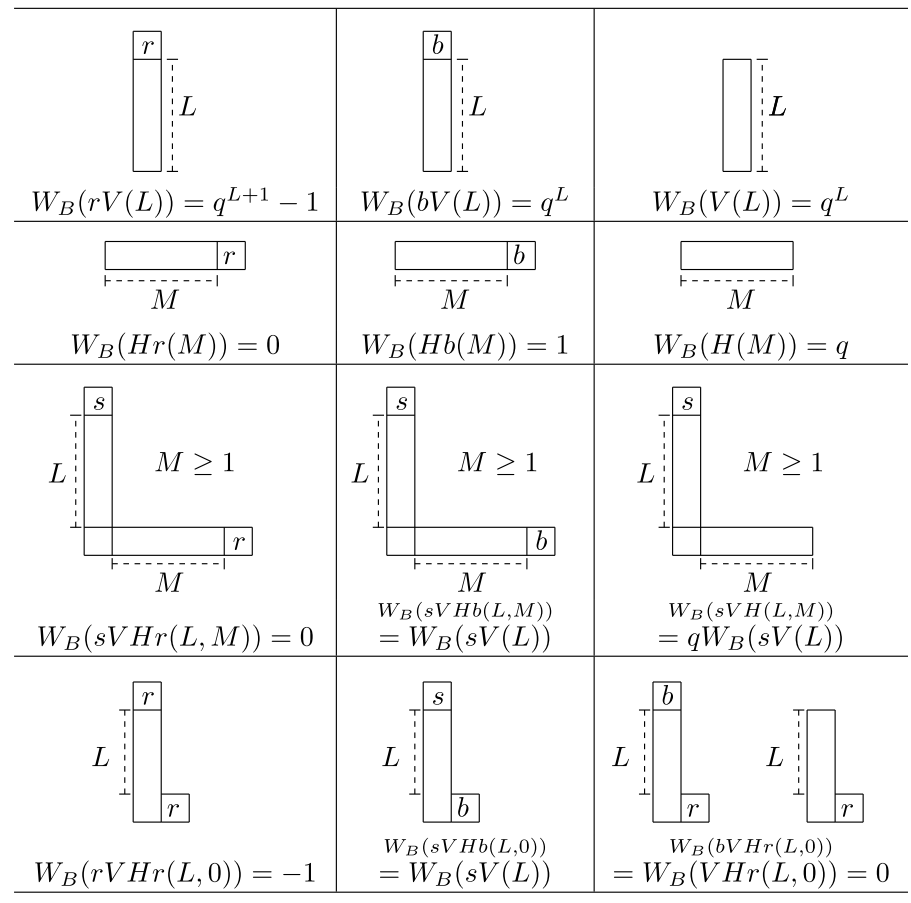

Remark 4.4. Table 1 is the summary of the weights of simple shape connecting rim hooks described in Lemmas 4.1, 4.2 and Proposition 4.3.

Finally, we consider a general connecting $\operatorname{rim}$ hook $G$ which consists of a hook shape $\operatorname{VHt}\left(L_{0}, M_{0}\right)$ where we have attached series of hook shapes $V H\left(L_{i}, M_{i}\right)$ for $i=1, \ldots, k-1$, and finally attached $s V H\left(L_{k}, M_{k}\right)$, a hook shape, as pictured in Figure 9. Here we assume that $k \geq 1$.

Note that for any statement $A$, we let $\chi(A)=1$ if $A$ is true and $\chi(A)=0$ if $A$ is false.

Proposition 4.5. For a rim hook $G$ in Figure 9,

$$
\begin{aligned}
W_{B}(G) & =W_{B}\left(s V\left(L_{k}\right)\right) W_{B}\left(V H t\left(L_{0}, M_{0}\right)\right) \prod_{i=1}^{k-1} W_{B}\left(V\left(L_{i}\right)\right) \\
& =\chi(t \neq r) q^{\chi(t=b l a n k)} W_{B}\left(s V\left(L_{k}\right)\right) q^{\sum_{i=0}^{k-1} L_{i}}
\end{aligned}
$$

where $W_{B}\left(s V\left(L_{k}\right)\right)$ and $W_{B}\left(V\left(L_{i}\right)\right)$ are as defined in Lemma 4.1 and $W_{B}\left(\operatorname{VHt}\left(L_{0}, M_{0}\right)\right)$ is as defined in Proposition 4.3. 


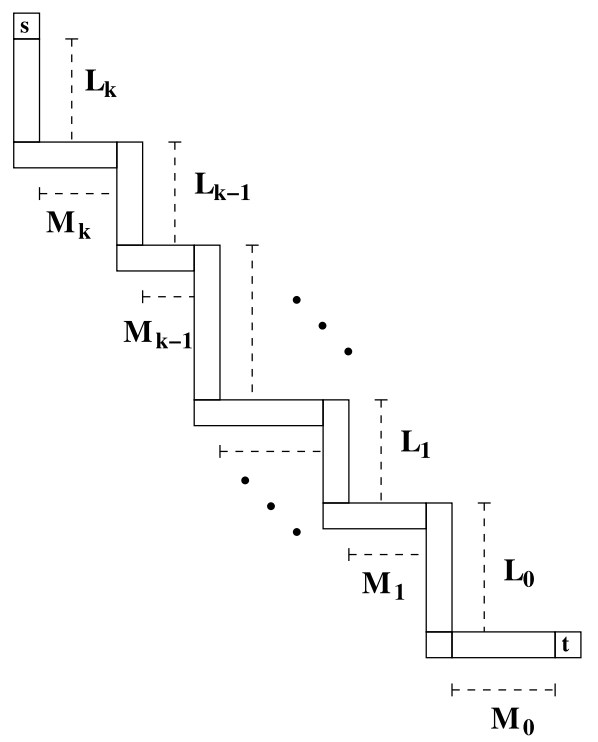

Figure 9: A general connecting rim hook $G$.

Proof. Since the set of $r b$-colored cells must form a vertical strip, the set of configurations over we must sum the weights is completely determined by how many $r b$ cells lie in each of the vertical strips $L_{i}$ for $i=1, \ldots k$ plus how many $r b$ cells lie in the lowest hook $V H t\left(L_{0}, M_{0}\right)$. It easily follows that

$$
W_{B}(G)=W_{B}\left(s V\left(L_{k}\right)\right) W_{B}\left(V H t\left(L_{0}, M_{0}\right)\right) \prod_{i=1}^{k-1} W_{B}\left(V\left(L_{i}\right)\right) .
$$

Now it follows from Lemma 4.1 that $W_{B}\left(V\left(L_{i}\right)\right)=q^{L_{i}}$ for $i=1, \ldots k-1$ so that $\prod_{i=1}^{k-1} W_{B}\left(V\left(L_{i}\right)\right)=q^{\sum_{i=1}^{k-1} L_{i}}$. It follows from Proposition 4.3 that

(i) $W_{B}\left(V H t\left(L_{0}, M_{0}\right)\right)=0$ if $t=r$,

(ii) $W_{B}\left(V H t\left(L_{0}, M_{0}\right)\right)=q^{L_{0}}$ if $t=b$, and

(iii) $W_{B}\left(V H t\left(L_{0}, M_{0}\right)\right)=q q^{L_{0}}$ if $t=$ blank.

Thus

$$
W_{B}(G)=\chi(t \neq r) q^{\chi(t=\text { blank })} W_{B}\left(s V\left(L_{k}\right)\right) q^{\sum_{i=0}^{k-1} q^{L_{i}}}
$$

We now pause to collect some observations about weights of the connecting rim hooks based on Lemmas 4.1 and 4.2 and Propositions 4.3 and 4.5. The first thing to notice is that the weights of all $s-r$ connecting rim hooks 
The combinatorics of the HMZ operators applied to Schur functions 427

where $s \in\{$ blank, $r, b\}$ are zero except for connecting rim hooks of the form $r V H r(L, 0)$ which have weights -1 . This has a number of consequences for the computation of $\mathbb{B}_{m}\left(s_{\lambda}[X]\right)$ according to formula (4.12). For any given $\gamma$ such that $s_{\gamma}[X]$ appears on the right hand side of (4.12) with a nonzero coefficient, we know that cells of $\lambda / \gamma$ must be colored red and the cells of $\gamma / \lambda$ must be colored blue. Now we claim that we cannot have a blue cell in $\gamma / \lambda$ appear above a red cell in $\lambda / \gamma$ since otherwise we would have a $b-r$ connecting rim hook for $\gamma$. In such a case, the weight of that $b-r$ connecting rim hook would be zero and hence, by (4.13), the coefficient of $s_{\gamma}[X]$ in $\mathbb{B}_{m}\left(s_{\lambda}[X]\right)$ would be zero. Thus the only $s_{\gamma}[X]$ that have nonzero coefficients in the expansion of $\mathbb{B}_{m}\left(s_{\lambda}[X]\right)$ must arise by first removing a broken rim hook $H$ from $\lambda$ and then adding back a vertical strip $V$ of size $|H|+m$ below $H$ to form $\gamma$. Now if $H$ consists of rim hooks $\left(h_{1}, \ldots, h_{p}\right)$ reading from top to bottom, then for $i=1, \ldots, p-1$, we claim that it must also be the case that the connecting rim hooks between the bottom cell of $h_{i}$ and the top cell $h_{i+1}$ must be of the form $r V H r\left(L_{i}, 0\right)$ for some $L_{i}$. That is, it also follows from Propositions 4.3 and 4.5 that the only $r-r$ connecting rim hook that have nonzero weight are connecting rim hooks of the form $r V H r\left(L_{i}, 0\right)$ which have weight -1 . Thus for $s_{\gamma}[X]$ that have nonzero coefficient in the expansion of $\mathbb{B}_{m}\left(s_{\lambda}[X]\right)$, it must be the case that if the bottom cell of $h_{i}$ lies in column $s_{i}$, then the top cell of $h_{i+1}$ must lie in column $s_{i}+1$ for $i=1, \ldots, p-1$. In addition, $h_{1}$ must start in column 1 since otherwise we would have a blank-r connecting rim hook and the weight of all such connecting rim hooks are zero. Thus we have the following theorem.

Theorem 4.6.

$$
\mathbb{B}_{m} s_{\lambda}[X]=\sum_{\gamma \vdash(n+m)} b_{m, \lambda, \gamma}(q) s_{\gamma}[X],
$$

where $b_{m, \lambda, \gamma}(q)=0$ unless

(1) $\gamma$ arises by adding a vertical strips of size $m$ on the outside of $\lambda$ or

(2) $\gamma$ arises from $\lambda$ by first removing a nonempty broken rim hook $H=\lambda / \mu$ of $\lambda$ from $\lambda$ which starts in the first column to get a partition $\mu$ and then adding a vertical strip $V$ of size $m+|\lambda / \mu|$ on the outside of $\mu$ to obtain $\gamma$ so that all the cells of $H$ lie strictly above all the cells of $V$. Moreover, if $H$ consists rim hooks $\left(h_{1}, \ldots, h_{p}\right)$, reading from top to bottom, then for $i=1, \ldots, p-1$, if the bottom cell of $h_{i}$ lies in column $s_{i}$, then the top cell of $h_{i+1}$ must lie in column $s_{i}+1$.

Next consider the shapes $\gamma$ that satisfy either condition (1) or (2) of Theorem 4.6. If $\gamma$ satisfies condition (1), then the only connecting rim hooks 
of $\gamma$ will be either blank- $b$ connecting rim hooks, $b-b$ connecting rim hooks, or $b$-blank connecting rim hooks. For any connecting rim hook $c$, the let $h t(c)$ denote the height of $c$. It follows from Lemmas 4.1 and 4.2 and Propositions 4.3 and 4.5 that

(a) the weight of a blank- $b$ connecting rim hook is equal to $q^{h t(c)-1}$,

(b) the the weight of a $b-b$ connecting rim hook is equal to $q^{h t(c)-2}$, and

(c) the weight of a $b$-blank connecting rim hook is equal to $q^{h t(c)-1}$.

Blue squares that are not part of connecting rim hooks have weight 1 . Thus it follows from (a), (b), and (c) that if $\gamma$ arises from $\lambda$ by adding a vertical strip of size $m$ on the outside of $\lambda$, then $b_{m, \lambda, \gamma}=q^{\ell(\gamma)-m}$.

Next suppose that $\gamma$ meets condition (2) of Theorem 4.6. First consider the broken rim hook $H=\lambda / \gamma=\left(h_{1}, \ldots, h_{p}\right)$. For $i=1, \ldots, p-1$, the connecting rim hook which connects the bottom cell of $h_{i}$ to the top cell of $h_{i+1}$ is of the form $r V H r\left(L_{i}, 0\right)$ for some $L_{i}$ so that we know that the weight of each of these rim hooks is -1 by Proposition 4.3. Thus these connecting rim hooks contribute a factor of $(-1)^{p-1}$ to $b_{m, \lambda, \gamma}$. Since we are assuming that $m>0$ so that there will be blue squares in $\gamma$, it follows that the lowest red cell of $h_{p}$ is part of a $r-b$ a connecting rim hook, say $C$. Thus $C$ is either of the form $r V H b(L, M)$ or it is of the form of rim hook pictured in Figure 9 where $s=r$ and $t=b$. Let $u$ be the row of red cell in $C, v$ be the highest row which contains a cell in a column to the right of the red cell in $C$, and $z$ be the row of the blue cell at the end of $C$. If $C$ is of the form $r V H b(L, M)$, then $v=z$ and $L=u-v-1$. In that case, it follows from Proposition 4.3 that the weight contributed by $C$ is $q^{L+1}-1=q^{u-v}-1$. If $C$ is of the form of rim hook pictured in Figure 9 where $s=r$ and $t=b$, then $L_{k}=u-v-1$ and the weight of $C$ is $\left(q^{L_{k}-1}+1\right) q^{\sum_{i=0}^{k-1} L_{i}}=\left(q^{u-v}-1\right) q^{v-z}$. In either case, we can argue as we did for the shapes that satisfy condition (1) of Theorem 4.6 that the weight contributed by the $b$ - $b$ and $b$-blank connecting rim hooks that lie on or below row $z$ is $q^{z-|V|}$. It follows that in both cases, the factor contributed by all the connecting rim hooks that lie on or below row $u$ is $\left(q^{u-v}-1\right) q^{v-|V|}$. Thus if $\gamma$ satisfies condition (2) of Theorem 4.6, then the factor of the total weight contributed by the connecting rim hooks is $(-1)^{p-1}\left(q^{u-v}-1\right) q^{v-|V|}$. Combining these observations with Theorem 4.6 yields the following theorem.

Theorem 4.7. Suppose that $\lambda=\left(\lambda_{1}, \ldots, \lambda_{k}\right)$ is a partition of $n$ and $m>0$. Then

$$
\mathbb{B}_{m} s_{\lambda}[X]=\sum_{\gamma \vdash(n+m)} b_{m, \lambda, \gamma} s_{\gamma}[X],
$$


The combinatorics of the HMZ operators applied to Schur functions 429

where

(a) $b_{m, \lambda, \gamma}=0$ unless $\gamma$ satisfies either condition (1) or condition (2) of Theorem 4.6.

(b) $b_{m, \lambda, \gamma}=q^{\ell(\gamma)-m}$ if $\gamma$ satisfies condition (1) of Theorem 4.6, and

(c) if $\gamma$ satisfies condition (2) of Theorem 4.6 and $u$ is the height of the lowest red cell that was removed from $\lambda, v$ is the height of the highest row which contains a cell in $\gamma$ which is to right of the lowest red cell removed from $\lambda, R$ is the set of red cells in that were removed from $\lambda$ that are not in any connecting rim hook, and $p$ is the number of rim hooks that make up the broken rim hook $\lambda / \gamma$, then

$$
b_{m, \lambda, \gamma}=(-1)^{p-1}\left(q^{u-v}-1\right) q^{v-m-|\lambda / \gamma|} \prod_{c \in R} w_{B}(c)
$$

where

$$
w_{B}(s)= \begin{cases}-1 & \text { if s has a red cell to its right } \\ q & \text { if s has a red cell below it, and } \\ q-1 & \text { if s is the lowest cell of a rim hook }\end{cases}
$$

Note that if $m \geq \ell(\lambda)-1$, then we can not be in case (c) of Theorem 4.7. That is, the fact that the broken $\operatorname{rim} H=\lambda / \gamma$ must contain a cell in the first column means that $H$ must contain the entire top row of $\lambda$. Thus if $\lambda=\left(\lambda_{1} \geq \cdots \geq \lambda_{k}\right)$, then we could not add a vertical strip of size $m+|H| \geq$ $k-1+\lambda_{1}$ below the lowest cell in $H$. Thus we have the following corollary.

Corollary 4.8. Suppose that $\ell(\lambda)=k$. Then for all $m \geq k-1$,

$$
\mathbb{B}_{m}\left(s_{\lambda}[X]\right)=\sum_{\substack{\lambda \subseteq \gamma \\ \gamma / \lambda \text { is a vertical strip }}} q^{\ell(\gamma)-m} s_{\gamma}[X] .
$$

Example 4.9. Suppose that we want to compute $\mathbb{B}_{2}\left(s_{\lambda}[X]\right)$ where $\lambda=$ $\left(3,2,1^{2}\right)$. Then in the first two rows of Figure 10, we have listed the 8 shapes that arise by adding a vertical strip of size 2 on the outside of $\lambda$. By Theorem $4.7, b_{2, \lambda, \gamma}=q^{\ell(\gamma)-2}$ for each of these shapes so we have written $b_{2, \lambda, \gamma}$ below each of these shape. There is only one shape $\gamma$ that falls in case (c) of Theorem 4.7 which is pictured at the end for row 2 in Figure 10. In this, $b_{2, \lambda, \gamma}=q-1$. Thus

$$
\begin{aligned}
\mathbb{B}_{2}\left(s_{\left(3,2,1^{2}\right)}[X]\right) & \\
= & q^{4} s_{\left(3,2,1^{4}\right)}+q^{3} s_{\left(3,2^{2}, 1^{2}\right)}+q^{3} s_{\left(3^{2}, 1^{3}\right)}+q^{3} s_{\left(4,2,1^{3}\right)}+q^{2} s_{\left(3,2^{3}\right)} \\
& +q^{2} s_{\left(3^{2}, 2,1\right)}+q^{2} s_{\left(4,2^{2}, 1\right)}+q^{2} s_{\left(4,3,1^{2}\right)}+(q-1) s_{(4,3,2)} .
\end{aligned}
$$



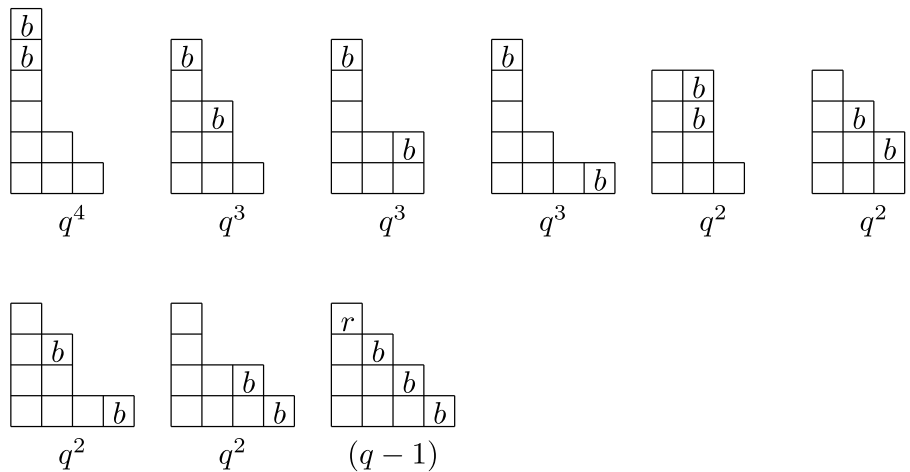

Figure 10: Shapes which occur in the expansion of $\mathbb{B}_{2}\left(s_{(3,2,1,1)}\right)$.

Example 4.10. For $n \leq m$,

$$
\mathbb{B}_{m}\left(\mathbb{B}_{n}(1)\right)=\mathbb{B}_{m}\left(s_{1^{n}}\right)=\mathbb{B}_{m}(\square n) .
$$

By Theorem $4.7, \mathbb{B}_{m}$ adds a vertical strip of size $m$ to the vertical strip of size $n$ in all possible ways. According to Theorem 4.7,

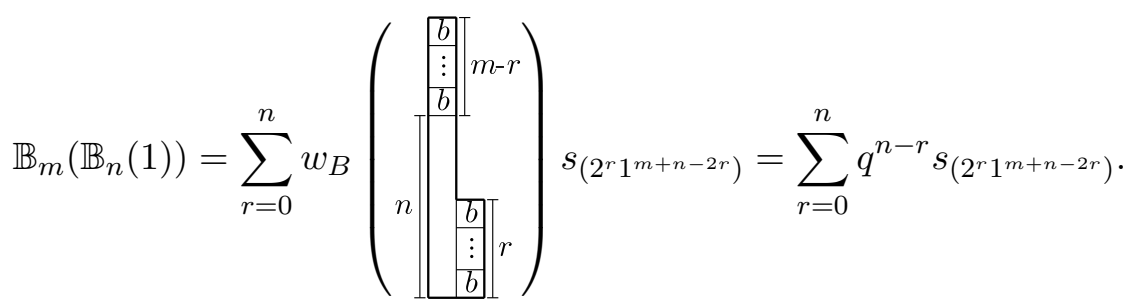

Similarly, $\mathbb{B}_{m+1}\left(\mathbb{B}_{n-1}(1)\right)=\sum_{r=0}^{n-1} q^{n-1-r} s_{\left(2^{r} 1^{m+n-2 r}\right)}$. Thus,

$$
\begin{aligned}
& \mathbb{B}_{m} \mathbb{B}_{n}(1)-q \mathbb{B}_{m+1} \mathbb{B}_{n-1}(1) \\
& \quad=\sum_{r=0}^{n} q^{n-r} s_{\left(2^{r} 1^{m+n-2 r}\right)}-\sum_{r=0}^{n-1} q^{n-r} s_{\left(2^{r} 1^{m+n-2 r}\right)}=s_{\left(2^{r} 1^{m+n-2 r}\right)} .
\end{aligned}
$$

Another way to compute $\mathbb{B}_{m} \mathbb{B}_{n}(1)$ is by recursion (4.5). That is, since $\mathbb{B}_{m}(1)=e_{m}=s_{1^{m}}$, then 
The combinatorics of the HMZ operators applied to Schur functions 431

$$
\begin{aligned}
\mathbb{B}_{m} \mathbb{B}_{n}(1) & =\mathbb{B}_{m}\left(e_{n}\right)=e_{n} e_{m}+(q-1) \sum_{j=1}^{n} q^{j-1} e_{n-j} \mathbb{B}_{m+j}(1) \\
& =e_{n} e_{m}+(q-1) \sum_{j=1}^{n} q^{j-1} e_{n-j} e_{m+j} .
\end{aligned}
$$

If we use the Pieri rule to calculate $e_{n-j} e_{m+j}=s_{1^{n-j}} s_{1^{m+j}}$, then it is not difficult to see that we get the same Schur function expansion but this is a much less efficient way to compute the Schur function expansion of $\mathbb{B}_{m} \mathbb{B}_{n}(1)$.

\section{Computing $\mathbb{C}_{a} s_{\lambda}[X]$}

There are two ways to approach the problem of computing $\mathbb{C}_{a} s_{\lambda}[X]$. One is to use the fact that $\mathbb{C}_{m}=(-1 / q)^{m-1} \omega \mathbb{B}_{m}^{q \rightarrow 1 / q} \omega$. Thus we can start with $s_{\lambda}[X]$, then compute $\mathbb{B}_{m} s_{\lambda^{\prime}}$ and replace $q$ by $1 / q$, then apply the $\omega$ transformation to the answer and finally multiply by $(-1 / q)^{m-1}$. This approach will not quite give the answer given in the introduction. The reason is that our notion of connecting rim hook which may include a top colored but not the bottom colored cell will change when we go the transpose shape $\lambda^{\prime}$ since the top colored cell will become the bottom colored cell which is not considered as a part of a connecting rim hook. Hence the result would have the discrepancy of the weights of those cells. The second approach, which is the one that we will follow in this section, is to show that arguments which are similar to those in the previous section will allow to explicitly compute the coefficients $c_{m, \lambda, \gamma}$ in terms of weight of connecting rim hooks as defined in the previous section.

\section{1. $\mathbb{C}_{m}$ applied to a skew Schur function}

We start by applying $\mathbb{C}_{m}$ to a skew Schur function $s_{\lambda / \nu}[X]$.

$$
\begin{gathered}
(-q)^{m-1} \mathbb{C}_{m} s_{\lambda / \nu}[X]=\left.s_{\lambda / \nu}\left[X-\frac{1-1 / q}{z}\right] \Omega[z X]\right|_{z^{m}} \\
=\left.\sum_{\nu \subseteq \mu \subseteq \lambda} s_{\mu / \nu}[X] s_{\lambda / \mu}\left[\frac{1 / q-1}{z}\right] \Omega[z X]\right|_{z^{m}}
\end{gathered}
$$




$$
\begin{aligned}
& =\left.\sum_{\nu \subseteq \mu \subseteq \lambda} s_{\mu / \nu}[X]\left(\frac{1}{q z}\right)^{|\lambda / \mu|} s_{\lambda / \mu}[1-q]\left(\sum_{n \geq 0} z^{n} h_{n}[X]\right)\right|_{z^{m}} \\
& =\sum_{\nu \subseteq \mu \subseteq \lambda} s_{\mu / \nu}[X]\left(\frac{1}{q z}\right)^{|\lambda / \mu|}\left(\sum_{\mu \subseteq \beta \subseteq \lambda} s_{\lambda / \beta}[1](-1)^{|\beta / \mu|} s_{\beta^{\prime} / \mu^{\prime}}[q]\right) \\
& \times\left.\left(\sum_{n \geq 0} z^{n} h_{n}[X]\right)\right|_{z^{m}} \\
& =\sum_{\nu \subseteq \mu \subseteq \lambda}\left[\left(\frac{1}{q}\right)^{|\lambda / \mu|} s_{\mu / \nu}[X]\left(\sum_{\mu \subseteq \beta \subseteq \lambda} s_{\lambda / \beta}[1](-1)^{|\beta / \mu|} s_{\beta^{\prime} / \mu^{\prime}}[q]\right) h_{m+|\lambda / \mu|}[X]\right] \\
& =\sum_{\nu \subseteq \mu \subseteq \lambda} s_{\mu / \nu}[X] \underbrace{\left(\sum_{\mu \subseteq \beta \subseteq \lambda}\left(\frac{1}{q}\right)^{|\lambda / \beta|} s_{\lambda / \beta}[1](-1)^{|\beta / \mu|} s_{\beta^{\prime} / \mu^{\prime}}[1]\right)}_{(* *)} h_{m+|\lambda / \mu|}[X] .
\end{aligned}
$$

To evaluate $\left.{ }^{* *}\right)$, note that $s_{\beta / \mu}[1]=0$ unless $\lambda / \beta$ is a horizontal strip and if $\lambda / \beta$ is a horizontal strip, then $s_{\lambda / \beta}[1]=1$. Similarly, $s_{\beta^{\prime} / \mu^{\prime}}[1]=0$ unless $\beta / \mu$ is a vertical strip and if it is a vertical strip, then $s_{\beta^{\prime} / \mu^{\prime}}[1]=1$. Now suppose that $\mu$ arises from $\lambda$ by first removing a horizontal strip to obtain $\beta$ and then removing a vertical strip to obtain $\mu$. Thus $\lambda / \mu$ is a BRH. Then we can interpret the associated weights $(1 / q)^{|\lambda / \beta|}$ and $(-1)^{|\beta / \mu|}$ as placing -1 in each cell of the vertical strip $\beta / \mu$ and $1 / q$ in each cell of the horizontal strip $\lambda / \beta$. It follows that when $\lambda / \mu$ is a BRH of $\lambda$, we must weight the cells in $\lambda / \mu$ as follows:

(i) if $s$ is a cell of $\lambda / \mu$ which has a cell in $\lambda / \mu$ to its right, then $s$ must have a $1 / q$ in it,

(ii) if $s$ is a cell of $\lambda / \mu$ which has a cell in $\lambda / \mu$ below it, then $s$ must have a -1 in it, and

(iii) if $s$ is the lowest cell in one of the rim hooks of $\lambda / \mu$ so that it neither has a cell of $\lambda / \mu$ to its right nor below it, then $s$ can have either -1 or $1 / q$ in it.

Hence we get

$$
\mathbb{C}_{m} s_{\lambda}[X]=\left(-\frac{1}{q}\right)^{m-1} \sum_{\substack{\mu \subseteq \lambda, \lambda / \mu \text { is a BRH of } \lambda}} w_{C}(\lambda / \mu) s_{\mu}[X] h_{m+|\lambda / \mu|}[X]
$$


The combinatorics of the HMZ operators applied to Schur functions 433

where

$$
w_{C}(\lambda / \mu)=\prod_{s \in \lambda / \mu} w_{C, \lambda / \mu}(s),
$$

and for each cell $s \in \lambda / \mu$,

$$
w_{C, \lambda / \mu}(s)= \begin{cases}1 / q & \text { if } s \text { has a cell of } \lambda / \mu \text { to its right } \\ -1 & \text { if } s \text { has a cell of } \lambda / \mu \text { below it, and } \\ \frac{1-q}{q} & \text { if } s \text { is the lowest cell of a rim hook in } \lambda / \mu .\end{cases}
$$

\section{2. $\mathbb{C}_{m}$ applied to Schur functions}

Next we shall follow the same steps that we used to evaluate $\mathbb{B}_{m} s_{\lambda}[X]$ to evaluate $\mathbb{C}_{m} s_{\lambda}[X]$. First note that in (5.1), by the Pieri rule,

$$
s_{\mu}[X] h_{m+|\lambda / \mu|}[X]=\sum_{\substack{\mu \subseteq \gamma, \gamma / \mu \text { is a } \\ \text { horizontal strip of size } \\ m+|\lambda / \mu|}} s_{\gamma}[X] .
$$

Hence,

$$
\begin{aligned}
& \mathbb{C}_{m} s_{\lambda}[X]=\left(-\frac{1}{q}\right)^{m-1} \sum_{\mu \subseteq \lambda, \lambda / \mu \text { is a BRH of } \lambda} w_{C}(\lambda / \mu) s_{\mu}[X] h_{m+|\lambda / \mu|}[X] \\
& =\left(-\frac{1}{q}\right)^{m-1} \sum_{\substack{\mu \subseteq \lambda, \lambda / \mu \text { is a BRH of } \lambda \\
\mu \subseteq \gamma, \gamma / \mu \text { is a horizontal strip of size } \\
m+|\lambda / \mu|}} w_{C}(\lambda / \mu) s_{\gamma}[X],
\end{aligned}
$$

where

$$
w_{C}(\lambda / \mu)=\prod_{s \in \lambda / \mu} w_{C, \lambda / \mu}(s),
$$

and for each cell $s \in \lambda / \mu, w_{C, \lambda / \mu}(s)$ is as defined in (5.3).

As before, we shall consider the cells in the broken rim hook $\lambda / \mu$ as being colored red so we put $r$ in the removed cells in $\lambda$, and the cells in the horizontal strip of size $|\lambda / \mu|+m$ as being colored blue so we put $b$ in the added cells in $\gamma$. Then in the right hand side of (5.4), the red and blue cells completely determine $\lambda$ and $\gamma$, but there could be many $\mu$ 's that could give rise to the same $\lambda$ and $\gamma$. That is, for the cells in the rim hooks connecting colored cells, it could be that some of these cells were originally removed from $\lambda$ to form $\mu$ and then added back to form $\gamma$. Thus like in the case of (4.12), we must evaluate the weights of the connecting rim hooks that arise by considering all possible contributions to the connecting rim hooks as $\mu$ varies. 


\subsection{Weights of connecting rim hooks}

We use the same notations $r V(L), b V(L), V(L)$, see Figure 6, and $\operatorname{Hr}(L)$, $H b(L), H(L)$, see Figure 7, for the vertical and horizontal strips of length $L$. Our first two lemmas are straightforward calculations so we omit the details.

Lemma 5.1. For $L \geq 1$,

$$
\begin{aligned}
W_{C}(r V(L)) & =0, \\
W_{C}(b V(L)) & =1, \quad \text { and } \\
W_{C}(V(L)) & =1 / q .
\end{aligned}
$$

Lemma 5.2. For $L \geq 1$,

$$
\begin{aligned}
W_{C}(H r(L)) & =\frac{q^{L+1}-1}{q^{L}(q-1)}, \\
W_{C}(H b(L)) & =1 / q^{L}, \quad \text { and } \\
W_{C}(H(L)) & =1 / q^{L} .
\end{aligned}
$$

Proposition 5.3. Let $s V H t(L, M)$ denote a hook shape connecting rim hook connecting cells colored $s$ and $t$ as in Figure 8 . If $L \geq 1$,

$$
\begin{aligned}
W_{C}(s V H t(L, M)) & =W_{C}(s V(L)) \times W_{C}(H t(M)) \\
& = \begin{cases}0, & \text { if } s=r, \\
W_{C}(H t(M)), & \text { if } s=b, \\
\frac{1}{q} W_{C}(H t(M)), & \text { if } s=\text { blank. }\end{cases}
\end{aligned}
$$

If $L=0$, then

$$
W_{C}(\operatorname{sVHt}(L, M))= \begin{cases}-1, & \text { if } s=r, t=r, \\ 0, & \text { if } s=r, t=b, \text { or } t=\text { blank }, \\ W_{C}(H t(M)), & \text { if } s=b \text { or blank, }\end{cases}
$$

where $W_{C}(H t(M))$ is as defined in Lemma 5.2.

Proof. If $L \geq 1$, then since the blue cells must form a horizontal strip, the south-left corner cell $c$ cannot be colored $r b$. Hence, the weight of the hook $s V H t(L, M)$ is the product of the weight of the vertical part of length $L$ and the weight of the horizontal part of length $M$. So we get $W_{C}(s V H t(L, M))$ by combining Lemmas 5.1 and 5.2 .

Now, let $L=0$. If $s=r$ and $t=r$, then there could be $k$ cells colored $r b$ for any $0 \leq k \leq M+1$. Now if $0 \leq k \leq M$, the cell $s$ contributes a factor 
The combinatorics of the HMZ operators applied to Schur functions 435 of $\frac{q}{1-q}$ and if $k=M+1$, then cell $s$ contributes a factor -1 . Thus

$$
\begin{aligned}
W_{C}(r V H r(L, M)) & =\frac{1-q}{q}\left(\sum_{k=0}^{M}\left(\frac{1}{q}\right)^{k}\right)+(-1)\left(\frac{1}{q}\right)^{M+1} \\
& =\frac{1}{q^{M+1}}-1-\frac{1}{q^{M+1}} \\
& =-1
\end{aligned}
$$

If $s=r$ and $t=b$, then the only difference is that for $1 \leq k \leq M+1$, the rightmost $r b$ cell contributes a factor $\frac{q}{1-q}$ instead of $\frac{1}{q}$. Thus

$$
\begin{aligned}
W_{C}(r V H b(L, M)) & =\frac{1-q}{q}+\left(\frac{1-q}{q}\right)^{2} \sum_{k=1}^{M}\left(\frac{1}{q}\right)^{k-1}+(-1)\left(\frac{1}{q}\right)^{M}\left(\frac{1-q}{q}\right) \\
& =\left(\frac{1-q}{q}\right)\left(1+\frac{1}{q^{M}}-1-\frac{1}{q^{M}}\right) \\
& =0 .
\end{aligned}
$$

The same calculation can be applied to the case when $s=r$ and $t$ is blank.

If $s$ is either blue or blank, then the cell $c$ cannot be colored $r b$. Then the weight of the hooks $b V H t(L, M)$ and $V H t(L, M)$ is just the weight of the horizontal strip $H t(M)$. Hence, we get the result by Lemma 5.2 .

Remark 5.4. Table 2 is the summary of the weights of simple shape connecting rim hooks described in Lemmas 5.1, 5.2 and Proposition 5.3.

Finally, we consider a general connecting rim hook $H$ which consists of a hook shape $s V H\left(L_{0}, M_{0}\right)$ where we have attached series of hook shapes $V H\left(L_{i}, M_{i}\right)$ for $i=1, \ldots, k-1$ and finally attached a hook $V H t\left(L_{k}, M_{k}\right)$ as pictured in Figure 11. Here we assume that $k \geq 1$.

Proposition 5.5. For a rim hook $H$ as in Figure 11,

$$
\begin{aligned}
W_{C}(H) & =W_{C}\left(s V H\left(L_{0}, M_{0}\right)\right) W_{C}\left(H t\left(M_{k}\right)\right) \prod_{i=1}^{k-1} W_{C}\left(H\left(M_{i}\right)\right) \\
& =\chi(s \neq r)\left(\frac{1}{q}\right)^{\chi(s=b l a n k)} W_{C}\left(H t\left(M_{k}\right)\right)\left(\frac{1}{q}\right)^{\sum_{i=0}^{k-1} M_{i}}
\end{aligned}
$$

where $W_{C}\left(s V H\left(L_{0}, M_{0}\right)\right), W_{C}\left(H t\left(M_{k}\right)\right)$ and $W_{C}\left(H\left(M_{i}\right)\right)$, for $i=1, \ldots$, $k-1$, are as defined in Lemma 5.2 and Proposition 5.3. 
Table 2: Weights of connecting rim hooks

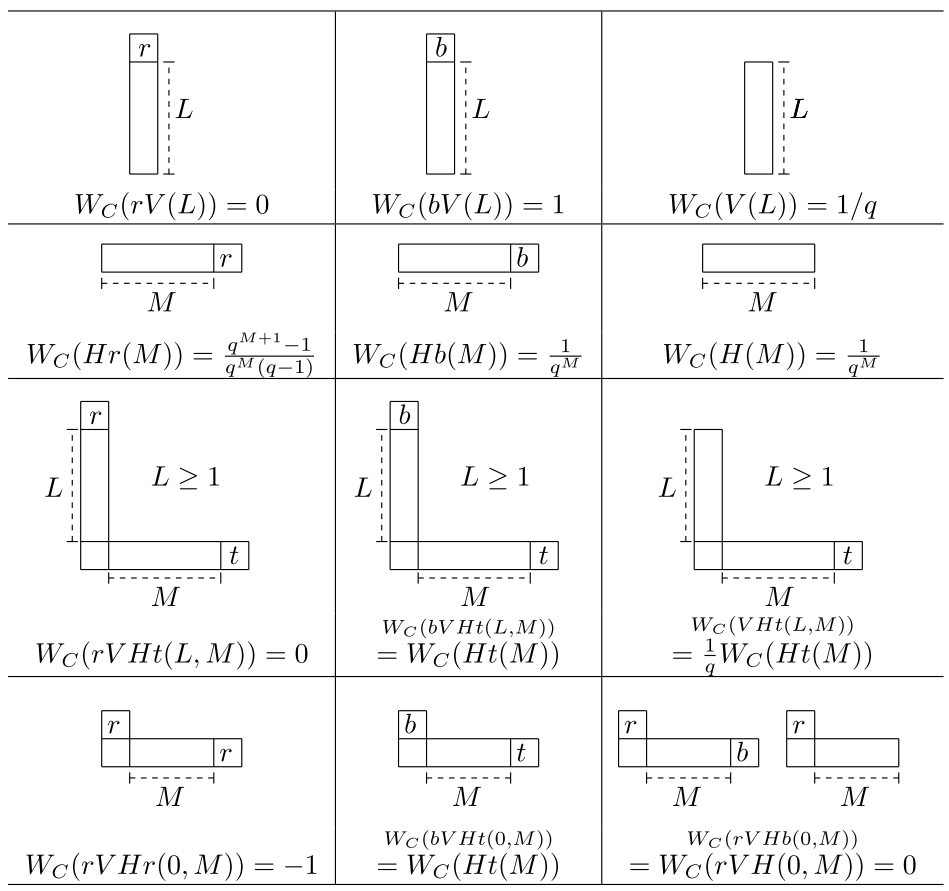

Proof. Since the set of $r b$ cells must form a horizontal strip, the set of configurations over we must sum the weights is completely determined by how many $r b$ cells lie in each of the horizontal strips $M_{i}$ for $i=1, \ldots, k$ plus how many horizontal cells lie in the top hook $s V H\left(L_{0}, M_{0}\right)$. Hence we easily get

$$
W_{C}(H)=W_{C}\left(s V H\left(L_{0}, M_{0}\right)\right) W_{C}\left(H t\left(M_{k}\right)\right) \prod_{i=1}^{k-1} W_{C}\left(H\left(M_{i}\right)\right) .
$$

It follows from Lemma 5.2 that $W_{C}\left(H\left(M_{i}\right)\right)=1 / q^{M_{i}}$ for $i=1, \ldots, k-1$ so $\prod_{i=1}^{k-1} W_{C}\left(H\left(M_{i}\right)\right)=(1 / q)^{\sum_{i=0}^{k-1} M_{i}}$. From Proposition 5.3,

(i) $W_{C}\left(s V H\left(L_{0}, M_{0}\right)\right)=0$ if $s=r$,

(ii) $W_{C}\left(s V H\left(L_{0}, M_{0}\right)\right)=\frac{1}{q^{M_{0}}}$ if $s=b$, and

(iii) $W_{C}\left(s V H\left(L_{0}, M_{0}\right)\right)=\frac{1}{q^{M_{0}+1}}$ if $s=$ blank.

Thus, we get

$$
W_{C}(H)=\chi(s \neq r)\left(\frac{1}{q}\right)^{\chi(s=\text { blank })} W_{C}\left(H t\left(M_{k}\right)\right)\left(\frac{1}{q}\right)^{\sum_{i=0}^{k-1} M_{i}} .
$$


The combinatorics of the HMZ operators applied to Schur functions 437

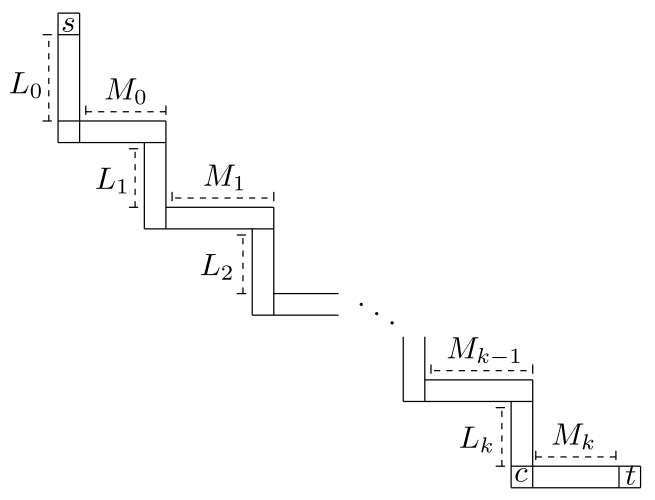

Figure 11: Any rim hook $H$.

Based on Lemmas 5.1, 5.2 and Propositions 5.3, 5.5, we can make a similar observation as we did with $\mathbb{B}_{m}$ operator. Note that the weights of all $r-s$ connecting rim hooks where $s \in\{$ blank, $r, b\}$ are zero except for connecting rim hooks of the form $r V H r(0, M)$ which have weight -1 . From Propositions 5.3 and 5.5, we can see that we cannot have a red cell in $\lambda / \gamma$ above a blue cell in $\gamma / \lambda$ since it would make the coefficient of $s_{\gamma}[X]$ in the expansion (5.4) zero. Thus we only have $s_{\gamma}[X]$ 's in the expansion of $\mathbb{C}_{m}\left(s_{\lambda}[X]\right)$ which arise by first removing a broken rim hook $H$ from $\lambda$ and then adding a horizontal strip $S$ of size $|H|+m$ above $H$ to form $\gamma$. Now let's say that $H=\lambda / \gamma$ consists of rim hooks $\left(h_{1}, \ldots, h_{p}\right)$ reading from bottom to top. Then for $i=1, \ldots, p-1$, we claim that it must be the case that the connecting rim hooks between the top cell of $h_{i}$ and the bottom cell of $h_{i+1}$ must be of the form $r \operatorname{VHr}\left(0, M_{i}\right)$ for some $M_{i}$ by Propositions 5.3 and 5.5. Thus for $s_{\gamma}[X]$ that have nonzero coefficient in the expansion of $\mathbb{C}_{m}\left(s_{\lambda}[X]\right)$, it must be the case that if the top cell of $h_{i}$ lies in row $r_{i}$, then the bottom cell of $h_{i+1}$ must lie in row $r_{i}+1$ for $i=1, \ldots, p-1$. Moreover, $h_{1}$ must start in row 1 since otherwise we would have a $r$-blank connecting rim hook which has a zero weight. Thus we derive the following theorem.

Theorem 5.6.

$$
\mathbb{C}_{m} s_{\lambda}[X]=\sum_{\gamma \vdash(n+m)} c_{m, \lambda, \gamma}(q) s_{\gamma}[X],
$$

where $c_{m, \lambda, \gamma}(q)=0$ unless

(1) $\gamma$ arises by adding a horizontal strip of size $m$ on the outside of $\lambda$, or

(2) $\gamma$ arises from $\lambda$ by first removing a nonempty broken rim hook $H=\lambda / \mu$ of $\lambda$ from $\lambda$ which starts in the first row to get a partition $\mu$ and then 
adding a horizontal strip $S$ of size $m+|\lambda / \mu|$ on the outside of $\mu$ to obtain $\gamma$ so that all the cells of $H$ lie strictly below all the cells of $S$. Moreover, if $H$ consists of rim hooks $\left(h_{1}, \ldots, h_{p}\right)$, reading from bottom to top, then for $i=1, \ldots, p-1$, if the top cell of $h_{i}$ lies in row $r_{i}$, then the bottom cell of $h_{i+1}$ must lie in row $r_{i}+1$.

Now we consider the shapes of $\gamma$ that satisfy either condition (1) or (2) of Theorem 5.6. If $\gamma$ satisfies condition (1), then the only connecting rim hooks of $\gamma$ would be either blank- $b$ connecting rim hooks, $b-b$ connecting rim hooks, or $b$-blank connecting rim hooks. For those connecting rim hooks, the horizontal cells which are not colored $b$ would contribute $1 / q$, based on Lemmas 5.1, 5.2 and Propositions 5.3, 5.5. Thus, we can see that if $\gamma$ arises from $\lambda$ by adding a horizontal strip of size $m$ on the outside of $\lambda$, then $c_{m, \lambda, \gamma}=(-1 / q)^{m-1}(1 / q)^{\gamma_{1}-m}=(-1)^{m-1}(1 / q)^{\gamma_{1}-1}$.

Next suppose that $\gamma$ satisfies condition (2) of Theorem 5.6. We first consider the broken rim hook $H=\lambda / \gamma=\left(h_{1}, \ldots, h_{p}\right)$. For $i=1, \ldots, p-1$, the connecting rim hook which connects the top cell of $h_{i}$ to the bottom cell of $h_{i+1}$ is of the form $r V \operatorname{Hr}\left(0, M_{i}\right)$ and we know that the weight of each of these rim hooks is -1 by Proposition 5.5. Thus these connecting rim hooks contribute a factor of $(-1)^{p-1}$ to $c_{m, \lambda, \gamma}$. By the condition (2) in Theorem 5.6, all the $r$ cells lie strictly below the $b$ cells, hence there would be only one connecting rim hook, say $C$, which is either of the form $b \operatorname{VHr}(L, M)$ or of the form of rim hook pictured in Figure 11 with $s=b$ and $t=r$. Let $u$ be the column of red cell at the end of $C, z$ be the column of the blue cell in $C$, and $v$ be the column of the lowest corner cell of $C$ which would be the cell $c$ in Figure 8 or Figure 11. If $C$ is of the form $b V \operatorname{Hr}(L, M)$, then $v=z$ and $M=u-z-1$. In this case, by Proposition 5.3, the weight of $C$ is

$$
W_{C}(H r(M))=\frac{q^{u-v}-1}{q^{u-z-1}(q-1)} .
$$

If $C$ is of the form of rim hook pictured in Figure 11 with $s=b$ and $t=r$, then $M_{k}=u-v-1$ and Proposition 5.5 gives that the weight of $C$ is

$$
\begin{aligned}
W_{C}\left(H r\left(M_{k}\right)\right)(1 / q)^{\sum_{i=0}^{k-1} M_{i}} & =\left(\frac{1}{q}\right)^{v-z} \frac{q^{u-v}-1}{q^{u-v-1}(q-1)} \\
& =\frac{q^{u-v}-1}{q^{u-z-1}(q-1)} .
\end{aligned}
$$

In both cases, the connecting rim hooks above $C$ are of the form $b$ - $b, b$-blank or blank- $b$. For those, as we did above for the shapes that satisfy condition (1), we can derive that the weight contribution would be $(1 / q)^{z-|S|}$. 
The combinatorics of the HMZ operators applied to Schur functions 439

Thus in both cases, the factor contributed by all the connecting rim hooks that lie before the column $u$ is $\frac{q^{u-v}-1}{q^{u-|S|-1}(q-1)}$. Hence, if $\gamma$ satisfies condition (2) of Theorem 5.6, then the factor of the total weight contributed by the connecting rim hooks would be

$$
(-1)^{p-1} \frac{q^{u-v}-1}{q^{u-|S|-1}(q-1)} .
$$

Combining these observations with Theorem 5.6 yields the following theorem.

Theorem 5.7. Suppose that $\lambda=\left(\lambda_{1}, \ldots, \lambda_{k}\right), \lambda_{1} \geq \cdots \geq \lambda_{k}$, is a partition of $n$ and $m>0$. Then

$$
\mathbb{C}_{m} s_{\lambda}[X]=\sum_{\gamma \vdash(n+m)} c_{m, \lambda, \gamma}(q) s_{\gamma}[X],
$$

where

(a) $c_{m, \lambda, \gamma}(q)=0$ unless $\gamma$ satisfies either condition (1) or condition (2) of Theorem 5.6,

(b) $c_{m, \lambda, \gamma}(q)=(-1)^{m-1}(1 / q)^{\gamma_{1}-1}$ if $\gamma$ satisfies condition (1) of Theorem 5.6, and

(c) if $\gamma$ satisfies condition (2) of Theorem 5.6 and $u$ is the column of the highest red cell that was removed from $\lambda, v$ is the column of the first corner cell to the left of the highest red cell of $\lambda, R$ is the set of red cells that were removed from $\lambda$ which are not in any connecting rim hooks, and $p$ is the number of rim hooks that make the broken rim hook $\lambda / \gamma$, then

$$
\begin{aligned}
c_{m, \lambda, \gamma}(q) & =\left(-\frac{1}{q}\right)^{m-1}(-1)^{p-1} \frac{q^{u-v}-1}{q^{u-m-|\lambda / \gamma|-1}(q-1)} \prod_{s \in R} w_{C}(s) \\
& =(-1)^{m+p} \frac{q^{u-v}-1}{q^{u-|\lambda / \gamma|-2}(q-1)} \prod_{s \in R} w_{C}(s)
\end{aligned}
$$

where

$$
w_{C}(s)= \begin{cases}1 / q & \text { if } s \text { has a red cell to its right } \\ -1 & \text { if s has a red cell below it, and } \\ \frac{1-q}{q} & \text { if } s \text { is the lowest cell of a rim hook }\end{cases}
$$


Note that if $m \geq \lambda_{1}-1$, then we cannot be the case (c) of Theorem 5.7. Thus we get the following corollary.

Corollary 5.8. Suppose that $\lambda_{1}=k$. Then for all $m \geq k-1$,

$$
\mathbb{C}_{m}\left(s_{\lambda}[X]\right)=\sum_{\substack{\lambda \subseteq \gamma \\ \gamma / \lambda \text { is a horizontal strip }}}\left(\frac{1}{q}\right)^{\gamma_{1}-1} s_{\gamma}[X]
$$

Example 5.9. For $b \geq a, n=a+b$,

$$
\mathbb{C}_{b}\left(\mathbb{C}_{a}(1)\right)=\mathbb{C}_{b}\left(\left(-\frac{1}{q}\right)^{a-1} s_{a}\right)=\left(-\frac{1}{q}\right)^{a-1} \mathbb{C}_{b}(\underbrace{\square}_{a})
$$

According to Theorem 5.7, to get the Schur expansion of $\mathbb{C}_{b}\left(s_{a}\right)$, we add a horizontal strip of size $b$ to the horizontal strip of size $a$. Then it would produce $\gamma=(a+b-r, r)$ shapes, for $0 \leq r \leq a$, and by Theorem 5.7 and Proposition 5.5, the coefficients of $s_{\gamma}$ would be $(-1 / q)^{n-2}(1 / q)^{a-r}$. Thus,

$$
\mathbb{C}_{b} \mathbb{C}_{a}(1)=\left(-\frac{1}{q}\right)^{n-2} \sum_{r=0}^{a}\left(\frac{1}{q}\right)^{a-r} s_{(n-r, r)}
$$

Note that we can carry out the same computation using (4.9). That is, $\mathbb{C}_{a}(1)=\left(-\frac{1}{q}\right)^{a-1} h_{a}$ so that

$$
\begin{aligned}
\mathbb{C}_{b} & \left(\left(-\frac{1}{q}\right)^{a-1} h_{a}\right) \\
& =\left(-\frac{1}{q}\right)^{a-1}\left(h_{a} \mathbb{C}_{b}(1)+(1-q) \sum_{j=1}^{a}(-1)^{j} h_{a-j} \mathbb{C}_{b+j}(1)\right) \\
& =\left(-\frac{1}{q}\right)^{a-1}\left(\left(-\frac{1}{q}\right)^{b-1} h_{a} h_{b}+(1-q) \sum_{j=1}^{a}(-1)^{j}\left(-\frac{1}{q}\right)^{b+j-1} h_{a-j} h_{b+j}\right) .
\end{aligned}
$$

One can then use the Pieri rule for the product of homogeneous symmetric functions to show that the right hand side of (5.9) reduces to (5.8) 
The combinatorics of the HMZ operators applied to Schur functions 441

but this is clearly a much less efficient way to calculate $\mathbb{C}_{b} \mathbb{C}_{a}(1)$ in this case.

Note that

$$
\mathbb{C}_{b+1} \mathbb{C}_{a-1}(1)=\left(-\frac{1}{q}\right)^{n-2} \sum_{r=0}^{a-1}\left(\frac{1}{q}\right)^{a-1-r} s_{(n-r, r)} .
$$

Hence,

$$
\begin{aligned}
q \mathbb{C}_{b} & \mathbb{C}_{a}(1)-\mathbb{C}_{b+1} \mathbb{C}_{a-1}(1) \\
& =\left(-\frac{1}{q}\right)^{n-2}\left[\sum_{r=0}^{a}\left(\frac{1}{q}\right)^{a-1-r} s_{(n-r, r)}-\sum_{r=0}^{a-1}\left(\frac{1}{q}\right)^{a-1-r} s_{(n-r, r)}\right] \\
& =\left(-\frac{1}{q}\right)^{n-2}\left(\frac{1}{q}\right)^{-1} s_{(b, a)} \\
& =(-1)^{n-2}\left(\frac{1}{q}\right)^{n-3} s_{(b, a)} .
\end{aligned}
$$

By applying $\mathbb{B}_{m}$ and $\mathbb{C}_{n}$ operators iteratively to 1 , we get a sum of two Schur functions with hook shapes.

Example 5.10.

$$
\begin{aligned}
& \mathbb{B}_{m}\left(\mathbb{C}_{n}(1)\right)=\mathbb{B}_{m}\left(\left(-\frac{1}{q}\right)^{n-1} s_{n}\right)=\left(-\frac{1}{q}\right)^{n-1} \mathbb{B}_{m}\left(s_{n}\right) \\
& =\left(-\frac{1}{q}\right)^{n-1}
\end{aligned}
$$

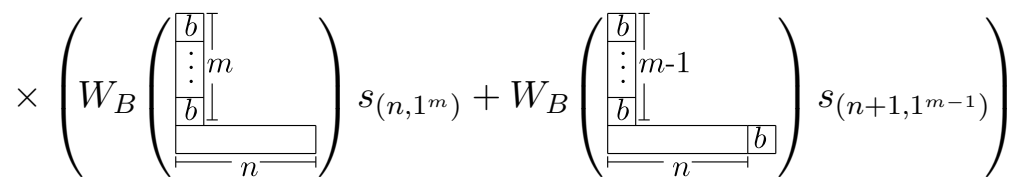

$$
\begin{aligned}
& =\left(-\frac{1}{q}\right)^{n-1}\left(q s_{\left(n, 1^{m}\right)}+s_{\left(n+1,1^{m-1}\right)}\right) \text {. }
\end{aligned}
$$

If we change the order of applying operators,

$$
\begin{aligned}
\mathbb{C}_{n}\left(\mathbb{B}_{m}(1)\right) & =\mathbb{C}_{n}\left(s_{1^{m}}\right) \\
& =\left(-\frac{1}{q}\right)^{n-1}
\end{aligned}
$$




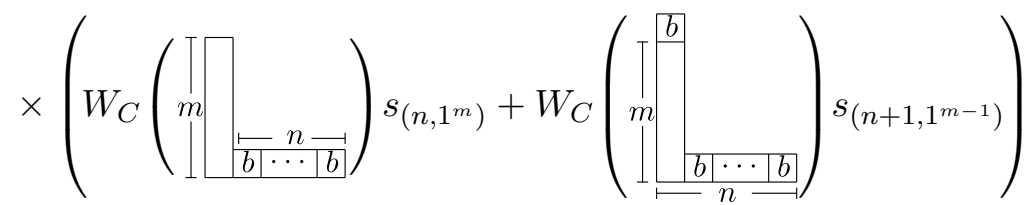

$$
\begin{aligned}
& =\left(-\frac{1}{q}\right)^{n-1}\left(s_{\left(n, 1^{m}\right)}+\frac{1}{q} s_{\left(n+1,1^{m-1}\right)}\right) \text {. }
\end{aligned}
$$

Thus we get

$$
\mathbb{B}_{m} \mathbb{C}_{n}(1)=q \mathbb{C}_{n} \mathbb{B}_{m}(1)
$$

We can use the combinatorial interpretation of Schur expansion of $\mathbb{C}_{m}\left(s_{\lambda}\right)$ in Theorem 5.7 to give a combinatorial proof of Propositions 3.2 and 3.3.

\section{Proposition 5.11.}

$$
e_{n}[X]=\sum_{\alpha \models n} C_{\alpha}[X ; q]
$$

Proof. We prove it by induction. If $n=1, \sum_{\alpha \models 1} C_{\alpha}[X ; q]=C_{1}[X ; q]=$ $h_{1}[X]=e_{1}[X]$. If $n>1$, then

$$
\begin{aligned}
& \sum_{\alpha \models n} C_{\alpha}[X ; q]=\sum_{\alpha \models n} \mathbb{C}_{\alpha_{1}}\left(\mathbb{C}_{\alpha_{2}} \cdots \mathbb{C}_{\alpha_{l(\alpha)}}(1)\right) \\
& =\sum_{r=0}^{n-1} \mathbb{C}_{n-r}\left(\sum_{\beta \models r} C_{\beta}[X ; q]\right) \\
& =\sum_{r=0}^{n-1} \mathbb{C}_{n-r}\left(e_{r}[X]\right)=\sum_{r=0}^{n-1} \mathbb{C}_{n-r}\left(s_{1^{r}}[X]\right) \\
& =\left(-\frac{1}{q}\right)^{n-1} s_{n}+\sum_{r=1}^{n-1}\left(-\frac{1}{q}\right)^{n-r-1}\left(\frac{1}{q} s_{\left(n-r+1,1^{r-1}\right)}+s_{\left(n-r, 1^{r}\right)}\right) \\
& =s_{1^{n}}[X]=e_{n}[X] \text {. }
\end{aligned}
$$

The second last line is by Theorem 5.7.

Proposition 5.12. For $0 \leq k<n$,

$$
s_{\left(n-k, 1^{n}\right)}[X]=(-q)^{n-k-1} \sum_{\substack{\alpha \models n \\ \alpha_{1} \geq n-k}} C_{\alpha}[X ; q]
$$


The combinatorics of the HMZ operators applied to Schur functions 443

Proof. When $n=1, k=0, s_{1}[X]=C_{1}[X ; q]$.

$$
\begin{aligned}
& \sum_{\substack{\alpha \in n \\
\alpha_{1} \geq n-k}} C_{\alpha}[X ; q] \\
& =\sum_{r=0}^{k} \mathbb{C}_{n-r}\left(\sum_{\alpha \models r} C_{\alpha}[X ; q]\right) \\
& \left.=\sum_{r=0}^{k} \mathbb{C}_{n-r}\left(s_{1^{r}}[X]\right) \quad \text { (by Proposition } 5.11\right) \\
& =\left(-\frac{1}{q}\right)^{n-1} s_{n}+\sum_{r=1}^{k}\left(-\frac{1}{q}\right)^{n-r-1}\left(\frac{1}{q} s_{\left(n-r+1,1^{r-1}\right)}+s_{\left(n-r, 1^{r}\right)}\right) \\
& =\left(-\frac{1}{q}\right)^{n-k-1} s_{\left(n-k, 1^{k}\right)} .
\end{aligned}
$$

Hence,

$$
(-q)^{n-k-1} \sum_{\substack{\alpha \models n \\ \alpha_{1} \geq n-k}} C_{\alpha}[X ; q]=s_{\left(n-k, 1^{k}\right)} .
$$

\section{A new recursion for the $q$-Kostka polynomials}

Recall that by (3.8),

$$
B_{\mu}[X ; q]=q^{n(\mu)} \omega \tilde{H}_{\mu}[X ; 0,1 / q]=\omega \sum_{\lambda} K_{\lambda \mu}(q) s_{\lambda}[X]=\sum_{\lambda} K_{\lambda \mu}(q) s_{\lambda^{\prime}}[X]
$$

where $K_{\lambda \mu}(q)=\sum_{T \in S S Y T(\lambda, \mu)} q^{\operatorname{ch}(T)}$. Note that since

$$
B_{\mu}[X ; q]=\mathbb{B}_{\mu_{1}} \mathbb{B}_{\mu_{2}} \cdots \mathbb{B}_{\mu_{l(\mu)}}(1),
$$

we can use our combinatorial interpretation of Schur expansion of $\mathbb{B}_{m}\left(s_{\lambda}\right)$ in Theorem 4.7 to give a completely different way to compute $K_{\lambda \mu}(q)$. This is illustrated in our next example.

Example 6.1. For $\mu=(2,1,1)$, one can compute using the charge statistic that

$$
\begin{aligned}
B_{(2,1,1)}[X ; q] & =\sum_{\lambda \vdash 4}\left(\sum_{T \in S S Y T\left(\lambda^{\prime}, \mu\right)} q^{c h(T)}\right) s_{\lambda}[X] \\
& =q^{3} s_{1^{4}}[X]+\left(q+q^{2}\right) s_{211}[X]+q s_{22}[X]+s_{31}[X] .
\end{aligned}
$$




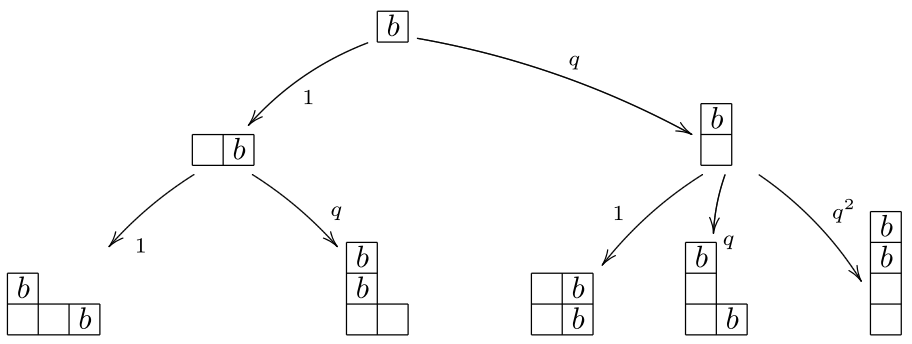

Figure 12: $B_{(2,1,1)}[X ; q]$ diagram.

On the other hand, we can construct $B_{(2,1,1)}[X ; q]$ by adding a vertical strip of size $\mu_{3}=1$ to 1 , and then adding vertical strips of size $\mu_{2}=1$ and $\mu_{1}=2$ to the results, iteratively. We can see the procedure in the diagram given in Figure 12. The weights on the branches are determined by the weights of connecting rim hooks which are specified in Theorem 4.7, and the coefficient $q^{\operatorname{ch}(T)}$ of $s_{\lambda}[X]$ can be calculated by multiplying all the weights on the branches connecting the diagram of $\lambda$ starting from the root.

This alternative way to compute the $K_{\lambda \mu}(q)$ also implies a new recursion for $q$-Kostka polynomials. That is, if $\lambda$ is a partition of $n$ and $\gamma$ is a partition of $n+m$, then we shall write $\lambda \rightsquigarrow \gamma$ if $\gamma$ satisfies the condition of Theorem 4.6, i.e. if either

(1) $\gamma$ arises by adding a vertical strips of size $m$ on the outside of $\lambda$ or

(2) $\gamma$ arises from $\lambda$ by first removing a nonempty broken rim hook $H=\lambda / \mu$ of $\lambda$ from $\lambda$ which starts in the first column to get a partition $\mu$ and then adding a vertical strip $V$ of size $m+|\lambda / \mu|$ on the outside of $\mu$ to obtain $\gamma$ so that all the cells of $H$ lie strictly above all the cells of $V$. Moreover, if $H$ consists rim hooks $\left(h_{1}, \ldots, h_{p}\right)$, reading from top to bottom, then for $i=1, \ldots, p-1$, if the bottom cell of $h_{i}$ lies in column $s_{i}$, then the top cell of $h_{i+1}$ must lie in column $s_{i}+1$.

Thus we can write Theorem 4.7 as

$$
\mathbb{B}_{m} s_{\lambda}[X]=\sum_{\lambda \rightsquigarrow \gamma} b_{m, \lambda, \gamma} s_{\gamma}[X] .
$$

Let $\mu=\left(\mu_{1} \geq \cdots \geq \mu_{l(\mu)}\right)$ be a partition of $n$ and let $\mu^{-}=\left(\mu_{2}, \mu_{3}, \ldots\right.$, $\left.\mu_{l(\mu)}\right)$. 
The combinatorics of the HMZ operators applied to Schur functions 445

Then the definition of $B_{\mu}[X ; q]$ implies that

$$
\begin{aligned}
B_{\mu}[X ; q] & =\mathbb{B}_{\mu_{1}} \mathbb{B}_{\mu_{2}} \cdots \mathbb{B}_{\mu_{l(\mu)}}(1) \\
& =\mathbb{B}_{\mu_{1}}\left(B_{\mu^{-}}[X ; q]\right)
\end{aligned}
$$

so that

$$
\begin{aligned}
B_{\mu}[X ; q] & =\sum_{\lambda \vdash n} K_{\lambda, \mu}(q) s_{\lambda^{\prime}}[X] \\
& =\mathbb{B}_{\mu_{1}}\left(B_{\mu^{-}}[X ; q]\right)=\mathbb{B}_{\mu_{1}}\left(\sum_{\eta \vdash\left(n-\mu_{1}\right)} K_{\eta, \mu^{-}}(q) s_{\eta^{\prime}}[X]\right) \\
& =\sum_{\eta \vdash\left(n-\mu_{1}\right)} K_{\eta, \mu^{-}}(q) \mathbb{B}_{\mu_{1}}\left(s_{\eta^{\prime}}[X]\right) \\
& =\sum_{\eta \vdash\left(n-\mu_{1}\right)} K_{\eta, \mu^{-}}(q) \sum_{\eta^{\prime} \leadsto \gamma} b_{\mu_{1}, \eta^{\prime}, \gamma} s_{\gamma}[X] \\
& =\sum_{\lambda^{\prime}} s_{\lambda^{\prime}}[X] \sum_{\substack{\eta \vdash\left(n-\mu_{1}\right) \\
\eta^{\prime} \leadsto \lambda^{\prime}}} b_{\mu_{1}, \eta^{\prime}, \lambda} K_{\eta, \mu^{-}}(q) .
\end{aligned}
$$

Thus we have the recursion

$$
K_{\lambda, \mu}(q)=\sum_{\substack{\eta \vdash n-\mu_{1} \\ \eta^{\prime} \rightsquigarrow \lambda^{\prime}}} b_{\mu_{1}, \eta^{\prime}, \lambda^{\prime}} K_{\eta, \mu^{-}}(q)
$$

For example, suppose $\lambda=(4,2)$ and $\mu=\left(2,1^{4}\right)$. Then to apply our recursion we must find the $\eta^{\prime}$ 's of size 4 such that $\eta^{\prime} \rightsquigarrow\left(2^{2}, 1^{2}\right)$. It is easy to see that there are three such $\eta^{\prime}$ s, namely, $(2,2),\left(2,1^{2}\right)$, and $\left(1^{4}\right)$. We have pictured these three shape at the left in Figure 13. In each case, $\lambda^{\prime}$ arises from $\eta^{\prime}$ by adding a vertical strip of size 2 to $\eta^{\prime}$ so that $b_{2, \eta^{\prime}, \lambda^{\prime}}=q^{\ell\left(\lambda^{\prime}\right)-2}=q^{2}$. Thus in this case, recursion (6.2) gives that

$$
K_{(4,2),\left(2,1^{4}\right)}(q)=q^{2} K_{(2,2),\left(1^{4}\right)}(q)+q^{2} K_{(3,1),\left(1^{4}\right)}(q)+q^{2} K_{(4),\left(1^{4}\right)}(q) .
$$

Next suppose that we want to compute $K_{(2,2),\left(1^{4}\right)}(q)$. Then we have to find the $\eta^{\prime}$ s of size 3 such that such that $\eta^{\prime} \rightsquigarrow(2,2)$. In this case, there are two such $\eta^{\prime}$ s, namely, $\left(1^{3}\right)$ and $(2,1)$. These are pictured on the right in Figure 13 along with the coloring of the squares which show how $\eta^{\prime} \rightsquigarrow(2,2)$. In this case, Theorem 4.7 gives that $b_{1,\left(1^{3}\right),(2,2)}=(q-1)$ and $b_{1,(1,2),(2,2)}=q$. Thus

$$
K_{(2,2),\left(1^{4}\right)}(q)=(q-1) K_{(3),\left(1^{3}\right)}(q)+q K_{(2,1),\left(1^{3}\right)}(q) .
$$



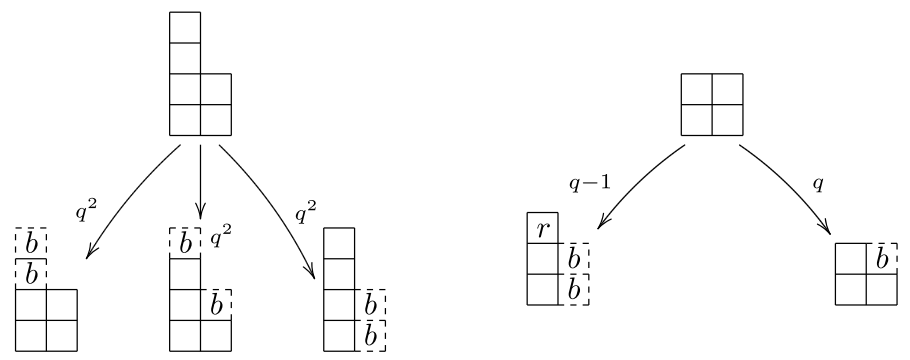

Figure 13: Examples of the $K_{\lambda, \mu}(q)$ recursion.

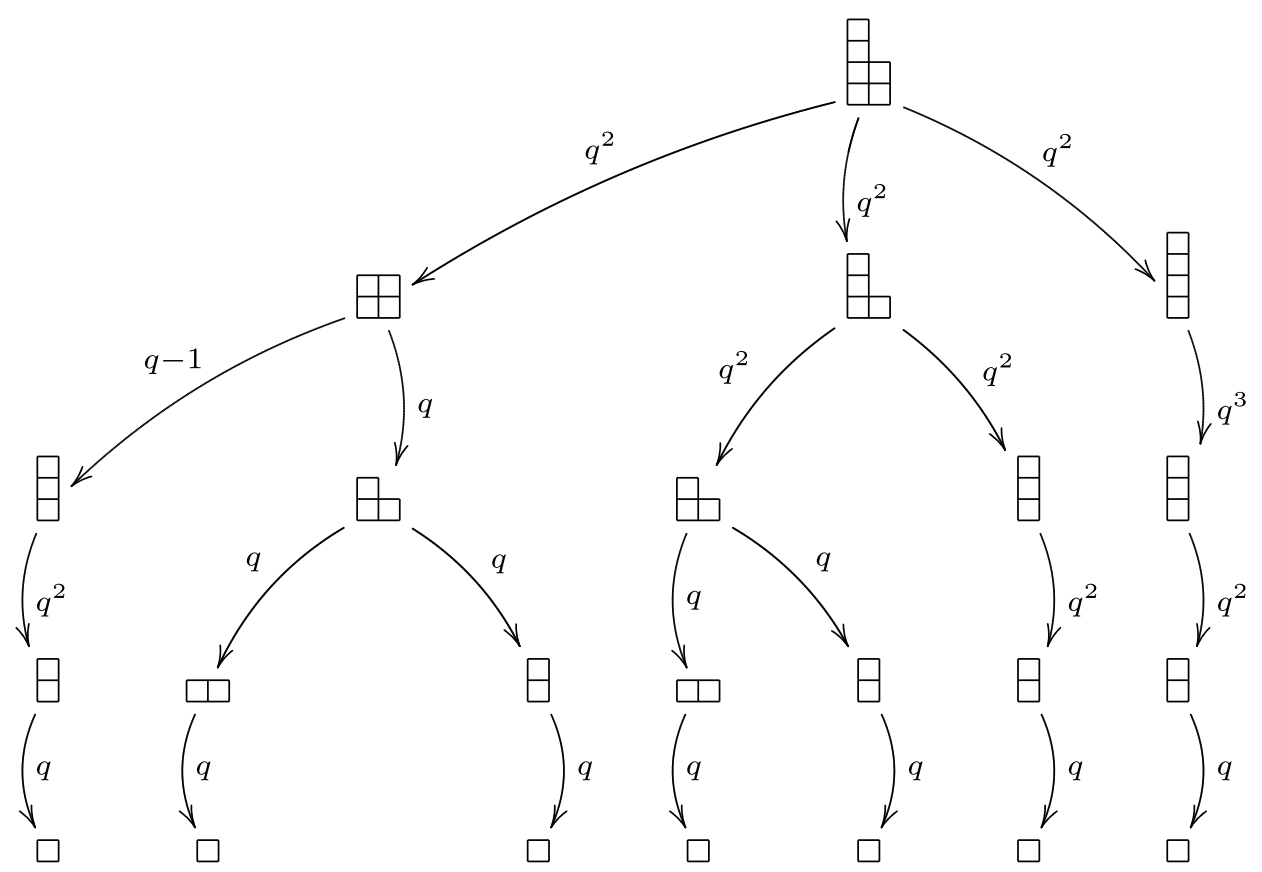

Figure 14: Charge tree to calculate $\sum_{T \in \operatorname{SSYT}\left(\lambda^{\prime}, \mu\right)} q^{\operatorname{ch}(T)}$ for $\mu=(2,1,1,1,1)$ and $\lambda^{\prime}=(2,2,1,1)$.

Note that since our recursion continually involves the conjugate shapes of the terms $K_{\lambda, \mu}(q)$ that we want to compute, we can construct a tree that represents the iteration of the our recursion to compute $K_{\lambda, \mu}(q)$. For example, to compute $K_{(4,2),\left(2,1^{4}\right)}(q)$, we would construct the tree pictured in Figure 14 where the weights on the branches are the weights of the corresponding $b_{m, \alpha, \beta}$. It then follows that $K_{(4,2),\left(2,1^{4}\right)}(q)$ is the sum of the product 
The combinatorics of the HMZ operators applied to Schur functions 447

\begin{tabular}{|c|c|c|c|c|c|}
\hline 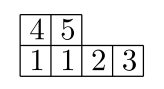 & \begin{tabular}{|l|l|l|l|}
3 & 5 & \multicolumn{2}{|c}{} \\
1 & 1 & 2 & 4 \\
\end{tabular} & \begin{tabular}{|l|l|l|l|}
3 & 4 & \multicolumn{2}{|c}{} \\
1 & 1 & 2 & 5 \\
\end{tabular} & 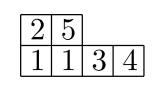 & \begin{tabular}{|l|l|l|l|}
2 & 4 & \multicolumn{1}{|c}{} \\
1 & 1 & 3 & 5 \\
\end{tabular} & \begin{tabular}{|l|l|l|l|}
2 & 3 & \multicolumn{2}{|c}{} \\
1 & 1 & 4 & 5 \\
\end{tabular} \\
\hline $\begin{array}{c}321154 \\
3_{2} 2_{1} 1_{0} 5_{3} 4_{2} \\
1_{0}\end{array}$ & $\begin{array}{c}42115 \\
4_{2} 2_{1} 1_{0} 5_{2} 3_{1} \\
1_{0}\end{array}$ & $\begin{array}{c}521143 \\
5_{3} 2_{1} 1_{0} 4_{2} 3_{1} \\
1_{0}\end{array}$ & $\begin{array}{c}431152 \\
4_{2} 3_{1} 1_{0} 5_{2} 2_{0} \\
1_{0}\end{array}$ & $\begin{array}{c}531142 \\
5_{2} 3_{1} 1_{0} 4_{1} 2_{0} \\
1_{0}\end{array}$ & $\begin{array}{c}541132 \\
5_{3} 4_{2} 1_{0} 3_{1} 2_{0} \\
1_{0}\end{array}$ \\
\hline$q^{8}$ & $q^{6}$ & $q^{7}$ & $q^{5}$ & $q^{4}$ & $q^{6}$ \\
\hline
\end{tabular}

Figure 15: Computing $K_{(4,2),\left(2,1^{4}\right)}(q)$ using the charge statistic.

of the weights along all the branches of this tree so that

$$
\begin{aligned}
K_{(4,2),\left(2,1^{4}\right)}(q) & =q^{5}(q-1)+q^{4}+q^{5}+q^{5}+q^{6}+q^{7}+q^{8} \\
& =q^{4}+q^{5}+2 q^{6}+q^{7}+q^{8} .
\end{aligned}
$$

To confirm the correctness of our calculation, we have pictured in Figure 15, the computation of $K_{(4,2),\left(2,1^{4}\right)}(q)$ as $\sum_{T \in \operatorname{SSYT}\left((2,4),\left(1^{4}, 2\right)\right)} q^{\operatorname{ch}(T)}$ using the definition of charge due to Lascoux and Schüzenburger [19]. In this case, amount of effort need to compute $K_{(4,2),\left(2,1^{4}\right)}(q)$ is about the same. One drawback of using recursion (6.2) is that it involves cancellation. Nevertheless, it is not difficult to find cases it requires less work to compute $K_{\lambda, \mu}(q)$ by building the recursion tree as we did in Figure 14 than computing $K_{\lambda, \mu}(q)$ by summing the charge of of all column strict tableaux of shape $\lambda$ and type $\mu$ and there are other cases where there is considerably less work in computing $K_{\lambda, \mu}(q)$ using the charge statistic as opposed to computing the recursion tree.

\section{References}

[1] N. Bergeron, F. Descouens and M. Zabrocki (2010). A filtration of $(q, t)-$ Catalan numbers. Adv. in Appl. Math. 44(1) 16-36. MR2552653

[2] F. Bergeron, A. M. Garsia, M. Haiman, and G. Tesler (1999). Identities and positivity conjectures for some remarkable operators in the theory of symmetric functions. Methods Appl. Anal. 6(3) 363-420. MR1803316

[3] E. S. Egge, J. Haglund, K. Killpatrick, and D. Kremer (2003). A Schröder generalization of Haglund's statistic on Catalan paths. Electron. J. Combin. 10(1). Research Paper 16, 21 pp. MR1975766 
[4] A. M. Garsia and J. Haglund (2001). A positivity result in the theory of Macdonald polynomials. Proc. Natl. Acad. Sci. USA 98(8) 4313-4316. MR1819133

[5] A. M. Garsia and J. Haglund (2002). A proof of the $q, t$-Catalan positivity conjecture. Discrete Math. 256(3) 677-717. LaCIM 2000 Conference on Combinatorics, Computer Science and Applications (Montreal, QC). MR1935784

[6] A. M. Garsia and M. Haiman (1996). A remarkable $q, t$-Catalan sequence and $q$-Lagrange inversion. J. Algebraic Combin. 5(3) 191-244. MR1394305

[7] I. M. Gessel (1983). Multipartite $P$-partitions and inner products of skew Schur functions. Combinatorics and Algebra (Boulder, Colo., 1983), Contemp. Math., 34 Amer. Math. Soc., Providence, RI, 1984, pp. 289-317. MR0777705

[8] J. Haglund (2003). Conjectured statistics for the $q, t$-Catalan numbers. Adv. Math. 175(2) 319-334. MR1972636

[9] J. Haglund, M. Haiman, N. Loehr, J. B. Remmel, and A. Ulyanov (2005). A combinatorial formula for the the character of the diagonal covariants, Duke Mathematical Journal 126(2) 195-232. MR2115257

[10] J. Haglund (2004). A proof of the $q, t$-Schröder conjecture. Internat. Math. Res. Notices 11 525-560. MR2038776

[11] J. Haglund and N. Loehr (2002). A conjectured combinatorial formula for the Hilbert series for diagonal harmonics. Proceedings of the 14th International Conference on Formal Power Series and Algebraic Combinatorics, Melbourne.

[12] J. Haglund, J. Morse and M. Zabrocki (2012). A compostional shuffle conjecture specifying touch points of the Dyck path. Canad. J. Math. 64 822-844. MR2957232

[13] M. Haiman (2002). Notes on Macdonald polynomials and the geometry of Hilbert schemes. Symmetric Functions 2001: Surveys of Developments and Perspectives, Proceeding of the NATO Advanced Study Institute. S. Fomin, ed. Kluwer, Dordrecht, pp. 1-64. MR2059359

[14] M. Haiman (1994). Conjectures on the quotient ring by diagonal invariants. J. Algebraic Combin. 3(1) 17-76. MR1256101 
The combinatorics of the HMZ operators applied to Schur functions 449

[15] M. Haiman (1998). $t, q$-Catalan numbers and the Hilbert scheme. Discrete Math.: Selected papers in honor of Adriano Garsia 193(1-3) 201224. MR1661369

[16] M. Haiman (2001). Hilbert schemes, polygraphs and the Macdonald positivity conjecture. J. Amer. Math. Soc. 14(4) 941-1006. MR1839919

[17] M. Haiman (2002). Vanishing theorems and character formulas for the Hilbert scheme of points in the plane. Invent. Math. 149 371-407. MR1918676

[18] N. H. Jing (1991). Vertex operators and Hall-Littlewood symmetric functions. Adv. Math. 87(2) 226-248. MR1112626

[19] A. Lascoux and M. P. Schützenberger (1978). Sur une conjecture do H. O. Foulkes. C.R. Acad. Sci. Paris Sér. A-B. 286(7) A323-A324. MR0472993

[20] N. Loehr and G. S. Warrington (2008). Nested quantum Dyck paths and $\nabla\left(s_{\lambda}\right)$. Int. Math. Res. Not. IMRN Art. ID rnm 157(5) 29 pp. MR2418288

[21] N. Loehr and J. B. Remmel (2004). Conjectured combinatorial models for the Hilbert series of generalized harmonics modules. Electronic $J$. of Combinatorics 11(1) R68, 64 pp. MR2097334

[22] N. Loehr and J. B. Remmel (2001). A computational and combinatorial exposé of plethysitic calculus. Journal of Algebraic Combinatorics 33(2) 163-198. MR2765321

[23] I. G. Macdonald (1995). Symmetric Functions and Hall Polynomials, 2nd ed. The Clarendon Press, Oxford University Press, New York. With contributions by A. Zelevinsky, Oxford Science Publications. MR1354144

[24] A. O. Morris (1963). The Characters of the group $G L(n, q)$. Math. Zeitschr. 81 112-123. MR0153750

[25] J. B. Remmel (2006). The Combinatorics of the Macdonald's $D_{n}^{1}$ operator. Seminaire Lotharingien de Combinatoire B54As 55 pp.

[26] M. P. Schützenberger (1977). La correspondance de Robinson. Combinatoire et représentation du groupe symétrique (Actes Table Ronde CNRS, Univ. Louis-Pasteur Strasbourg, Strasbourg, 1976, Springer, Berlin, Lecture Notes in Mathematics, Vol. 579, pp. 59-113. MR0498826 
[27] R. P. Stanley (1979). Invariants of finite groups and their applications to combinatorics. Bull. Amer. Math. Soc. (N.S.) 1(3) 475-511. MR0526968

[28] R. P. Stanley (1999). Enumerative Combinatorics. Vol. 2. Cambridge University Press, Cambridge. With a foreword by Gian-Carlo Rota and appendix 1 by Sergey Fomin. MR1676282

[29] D. W. Stanton and D. E. White (1985). A Schensted algorithm for rim hook tableaux. J. Combin. Theory Ser. A 40(2) 211-247. MR0814412

[30] M. Zabrocki. The action of the Hall-Littlewood vertex operator. Preprint. MR2697177

JEFFREY B. REMMEL

Department of Mathematics

UNIVERSITY OF CALIFORNIA

SAN Diego, CA 92093-0112

USA

E-mail address: jremmel@ucsd.edu

Meesue Yoo

School of Mathematics

Korea Institute for Advanced Study

South Korea

E-mail address: meesue@kias.re.kr

Received August 28, 2011 